\title{
The counter-propagating Rossby-wave perspective on baroclinic instability. II: Application to the Charney model
}

Article

Published Version

Heifetz, E., Methven, J., Hoskins, B. J. and Bishop, C. H. (2004) The counter-propagating Rossby-wave perspective on baroclinic instability. II: Application to the Charney model. Quarterly Journal of the Royal Meteorological Society, 130 (596). pp. 233-258. ISSN 1477-870X doi:

https://doi.org/10.1256/qj.02.185 (Part A) Available at https://centaur.reading.ac.uk/90/

It is advisable to refer to the publisher's version if you intend to cite from the work. See Guidance on citing.

Published version at: http://www.interscience.wiley.com

To link to this article DOI: http://dx.doi.org/10.1256/qj.02.185

Publisher: Royal Meteorological Society

All outputs in CentAUR are protected by Intellectual Property Rights law, including copyright law. Copyright and IPR is retained by the creators or other copyright holders. Terms and conditions for use of this material are defined in the End User Agreement. 


\section{CentAUR}

Central Archive at the University of Reading

Reading's research outputs online 


\title{
The counter-propagating Rossby-wave perspective on baroclinic instability.
} II: Application to the Charney model

\author{
By E. HEIFETZ ${ }^{1}$, J. METHVEN ${ }^{2 *}$, B. J. HOSKINS ${ }^{2}$ and C. H. BISHOP ${ }^{3}$ \\ ${ }^{1}$ Tel-Aviv University, Israel \\ ${ }^{2}$ University of Reading, $U K$ \\ ${ }^{3}$ Naval Research Laboratories/UCAR, Monterey, USA
}

(Received 10 September 2002; revised 12 June 2003)

\section{SUMMARY}

The constant-density Charney model describes the simplest unstable basic state with a planetary-vorticity gradient, which is uniform and positive, and baroclinicity that is manifest as a negative contribution to the potential-vorticity (PV) gradient at the ground and positive vertical wind shear. Together, these ingredients satisfy the necessary conditions for baroclinic instability. In Part I it was shown how baroclinic growth on a general zonal basic state can be viewed as the interaction of pairs of 'counter-propagating Rossby waves' (CRWs) that can be constructed from a growing normal mode and its decaying complex conjugate. In this paper the normal-mode solutions for the Charney model are studied from the CRW perspective.

Clear parallels can be drawn between the most unstable modes of the Charney model and the Eady model, in which the CRWs can be derived independently of the normal modes. However, the dispersion curves for the two models are very different; the Eady model has a short-wave cut-off, while the Charney model is unstable at short wavelengths. Beyond its maximum growth rate the Charney model has a neutral point at finite wavelength $(r=1)$. Thereafter follows a succession of unstable branches, each with weaker growth than the last, separated by neutral points at integer $r$-the so-called 'Green branches'. A separate branch of westward-propagating neutral modes also originates from each neutral point. By approximating the lower CRW as a Rossby edge wave and the upper CRW structure as a single PV peak with a spread proportional to the Rossby scale height, the main features of the 'Charney branch' $(0<r<1)$ can be deduced without prior calculation of the normal modes. Furthermore, CRWs from this branch are seen to make a smooth transition into the boundary and interior PV structure of the neutral modes appearing at $r=1$. The behaviour of the other branches and neutral points is essentially the same when viewed from the CRW perspective, but with cancelling interior PV structures reducing the self and mutual interaction of the CRWs. The underlying dynamics determining the nature of all the solutions is the difference in the scale-dependence of PV inversion for boundary and interior PV anomalies, the Rossby-wave propagation mechanism and the CRW interaction. The behaviour of the Charney modes and the first neutral branch, which rely on tropospheric PV gradients, are arguably more applicable to the atmosphere than modes of the Eady model where the positive PV gradient exists only at the tropopause.

\section{KEYWORDS: Dispersion relation Phase-locking Scale effect of PV inversion Steering level}

\section{INTRODUCTION}

The constant-density Charney model is the simplest baroclinic basic state whose linear dynamics cannot be explained by two edge waves. It has a negative temperature gradient at the surface in thermal wind balance with a linear profile of zonal wind with height. Meridional displacements of air at the surface result in surface temperature anomalies which can, in turn, induce circulation anomalies. In the absence of a potentialvorticity (PV) gradient in the interior of the fluid, a wave in surface temperature would propagate eastwards relative to the surface flow via the Rossby-wave mechanism as a neutral edge wave. However, the Charney model includes the planetary vorticity gradient $\beta$ which is positive and uniform throughout the interior of the model, enabling westward propagation of $\mathrm{PV}$ anomalies relative to the flow in the fluid interior. A surface temperature wave induces velocity which decays exponentially with height, influencing PV anomalies at all levels. Moreover, it is not possible to form a surface temperature anomaly by conservative displacement from a zonal state without also developing an interior PV anomaly near the surface with the opposite sign. Clearly it is not an exact

* Corresponding author: Department of Meteorology, University of Reading, PO Box 243, Earley Gate, Reading, Berkshire RG6 6BB, UK. e-mail: J.Methven@ reading.ac.uk

(c) Royal Meteorological Society, 2004. 
description to view baroclinic growth in the Charney model as the interaction of a pure surface temperature wave with an upper wave composed of interior PV anomalies at a single level.

Consider a normal-mode structure of the form $q^{\prime}=\operatorname{Re}\left\{q_{k} \mathrm{e}^{\mathrm{i} k(x-c t)}\right\}$, where $c$ can be a complex number, $c_{\mathrm{r}}+\mathrm{i} c_{\mathrm{i}}$. If its surface temperature anomalies dominate the interior $\mathrm{PV}$ in the inversion to obtain velocity near the ground, such a mode can be expected to have a 'steering level' located just above ground where its phase speed matches the zonal flow (i.e. $U(z)=c_{\mathrm{r}}$ ). Bretherton (1966a) showed that, in the limit $c_{\mathrm{i}} \ll c_{\mathrm{r}}$, if the meridional PV gradient is non-zero at the steering level, there is a meridional PV flux at this level that is independent of $c_{\mathrm{i}}$, while at the ground there is an opposite signed PV flux proportional to $c_{\mathrm{i}}$. He proved that the domain-integrated PV flux is zero, which implies that modes with steering levels above ground must be unstable $\left(c_{\mathrm{i}} \neq 0\right)$. In this case the height where $U(z)=c_{\mathrm{r}}$ was called the 'critical level' and the growth mechanism was described as 'critical-layer instability'. Lindzen et al. (1980) showed that the interior PV flux for Charney modes is centred on the critical level, but with a spread proportional to $c_{\mathrm{i}}$. They referred to the region where $c_{\mathrm{r}}-c_{\mathrm{i}}<U<c_{\mathrm{r}}+c_{\mathrm{i}}$ as the critical layer. Robinson (1989) extended this work by calculating the northward PV flux induced at one level by the PV at some other level. He found that the PV flux induced by surface temperature anomalies is strongly peaked above the steering level and rapidly decreases to zero below it. He also showed that this same structure indicates how the PV at each level contributes to the northward surface temperature flux and, therefore, argued that the mode growth can be described by the interaction between surface temperature and a thin layer of PV anomalies centred just above the steering level. This viewpoint is clearly related to the counter-propagating Rossby wave (CRW) perspective on baroclinic growth proposed by Bretherton (1966b) and discussed by Hoskins, McIntyre and Robertson (1985).

Lindzen et al. (1980) explained baroclinic instability in the Charney model in terms of the overreflection of vertically propagating Rossby waves. Central to this theory is a wave-trapping region at the critical level flanked by bounded wave-propagation regions above and below (Lindzen and Tung 1978). For constant-density flows, wave trapping occurs when $Q=\left(\beta-U_{z z} f_{0}^{2} / N^{2}\right) /\left(U-c_{\mathrm{r}}\right)-k^{2}<0$ and propagation when $Q>0$. Since $U-c_{\mathrm{r}}$ changes sign once across the critical layer, the bounds of wave-propagation regions are also determined by the sign of the PV gradient. The interior PV gradient is positive everywhere but, following Bretherton (1966a), the negative boundary temperature gradient can be replaced by a homogeneous boundary condition plus a $\delta$-function in negative PV gradient immediately above the ground. Wave overreflection only occurs in this infinitesimally small wave-propagation region with negative a PV gradient. Using this theory, the growth rate for a given wavelength can be estimated from the magnitude of the reflection coefficient (obtained by numerical calculation) and time taken for a Rossby wave to propagate back and forth across the overreflection region in the limit $c_{\mathrm{i}} \ll c_{\mathrm{r}}$. Since this method overestimates growth rates for all growing modes in the Charney model and this estimate depends upon behaviour at the critical level as $c_{\mathrm{i}} \rightarrow 0$, Lindzen et al. (1980) argued that critical-layer instability is responsible for the baroclinic growth of normal modes.

In the paper by Heifetz et al. (2003) (hereafter called Part I) it was shown that any initial conditions composed from a growing normal-mode solution and its decaying complex conjugate can be alternatively viewed as the propagation and interaction of two CRWs. Here, these two CRWs form the basis for arguments about the behaviour of the Charney-model solutions. The upper CRW has no surface signature and a tendency to propagate westward relative to the flow on the interior PV gradient, while the lower 
CRW is essentially an eastward propagating Rossby edge wave modified by partially cancelling contributions from its interior PV structure. From all the above perspectives on baroclinic instability, the surface temperature gradient plays a special role because it can be viewed as a negative PV gradient; elsewhere the PV gradient is positive but baroclinic instability is only possible if the PV gradient takes on opposite signs within the domain (Charney and Stern 1962). Furthermore, the Fjørtoft (1951) necessary condition for baroclinic instability, namely that the PV gradient and zonal wind should be positively correlated over the domain, is clearly related to the requirements that counter-propagation (zonally) should result in phase-locking for CRWs or, alternatively, to the conditions for vertical propagation of Rossby waves and their overreflection. Although an exact description of the Charney model cannot be obtained using CRWs without first calculating the normal-mode solution, it is shown that almost all of the features of the normal modes' dispersion relation and their structures can be explained by assuming simple CRW structures and using PV-thinking, CRW propagation and interaction arguments.

In section 2 a short summary of the CRW theory, derived in Part I of this paper, provides the essential arguments and equations used to discuss the Charney model. Some background details on the Charney model, including relevant scaling, are given in section 3. In section 4 we analyse the normal-mode solutions for the Charney model from the CRW perspective and conclude our results in section 5.

\section{CRW THEORY}

The main arguments and equations from the CRW theory that are the basis for explanations later are summarized here without derivation-refer to Part I for details. We consider linear conservative dynamics with balance, so that PV conservation and its inversion describe the evolution of perturbations to a specified baroclinic basic flow $U(y, z)$ :

$$
\left(\frac{\partial}{\partial t}+U \frac{\partial}{\partial x}\right) q^{\prime}+v^{\prime} \frac{\partial \bar{q}}{\partial y}=0 .
$$

For the Charney analysis we assume a quasi-geostrophic flow, where $q^{\prime}$ is the perturbation PV, $\bar{q}_{y}$ is the meridional PV gradient of the basic state and $v^{\prime}$ is the meridional geostrophic wind. The PV in (1) includes the boundary temperature distribution using the Bretherton (1966a) $\delta$-function technique, so that (1) applies on the boundaries as well. We use the Fourier series

$$
q^{\prime}=\sum q_{k} \mathrm{e}^{\mathrm{i} k x}, \quad v^{\prime}=-\mathrm{i} \sum v_{k} \mathrm{e}^{\mathrm{i} k x},
$$

where the $-\mathrm{i}$ on the right-hand side of (2b) ensures that $q_{k}$ and $v_{k}$ are real with the same sign for purely propagating structures whose positive PV anomalies are associated with cyclonic motion. Substituting $(2 \mathrm{a}, \mathrm{b})$ into (1) for a wave-like solution $\mathrm{e}^{\mathrm{i} k(x-c t)}$, then the Rossby-wave phase speed satisfies

$$
c=U-\frac{\gamma}{k}
$$

where $\gamma=\bar{q}_{y} v_{k} / q_{k}$ (hereafter we omit the suffix $k$ ). Since both $U$ and $\gamma$ are generally functions of $(y, z)$, so is $c$ and, thus, an initially untilted Rossby wave will be sheared apart in the absence of other disturbances. However, if two untilted Rossby waves exist together they can preserve their untilted structures and propagate coherently, but they will also make each other grow (or decay) and affect each other's phase speed. 
In Part I, two methods were presented for obtaining such a pair of untilted CRWs for disturbances that can be described by combining a single growing normal mode and its decaying complex conjugate. The 'home-base method' has the advantage that the evolution of such a disturbance can be fully described by considering the relative phase and amplitudes of the CRWs at only two locations in the meridional plane $(y, z)$, their home-bases, as depicted in Fig. 1 of Part I. It extends the two-level 'PVthinking' explanation for baroclinic instability (Bretherton 1966b) to general zonal flows. However, the method's weakness is that relevant home-bases for the waves must be anticipated from the basic state before the wave structures are calculated. It was shown that the two home-base choices determine the phase of each wave required to reconstruct the disturbance and that, once these choices are made, the CRW structures are also determined.

The second method is based on the global conservation of pseudomomentum and pseudoenergy. The CRW phases are uniquely determined by specifying orthogonality with respect to pseudomomentum and energy growth, and is therefore objective. The structures obtained for the CRWs can sometimes be used to infer their home-bases in such a way that the disturbance evolution can again be described by CRW interaction at only two locations using the results of the first method. However, in some situations (notably the Green ranges examined in section 4(d)) the home-bases inferred by the orthogonality constraints are not as useful for discussing baroclinic growth as other home-base choices. Therefore, it is necessary to illustrate the results of both methods applied to the Charney model.

\section{(a) The home-base method}

A simple way to construct an untilted wave is to subtract a growing normal-mode solution of (1) from its decaying complex conjugate, since their structures have opposite tilts. The resulting structure contains at least one zonal line of zero-PV signature. Repeating this process for a different phase difference between the pair of the modes yields another untilted structure whose zero-PV line is located elsewhere. Along the zonal line $\left(y_{1}, z_{1}\right)$ the PV only has contributions from the first wave, and the PV of wave- 2 is identically zero. This is described as the home-base of wave- 1 . Similarly the home-base of wave-2 is located where the PV of wave-1 is zero. Solving (1) on the two home-bases gives evolution equations for the wave amplitudes $\left(a_{1}, a_{2}\right)$ and phases $\left(\epsilon_{1}, \epsilon_{2}\right)$ :

$$
\begin{gathered}
\dot{a}_{1}=-\gamma_{2}^{1} a_{2} \sin \epsilon, \quad \dot{a}_{2}=\gamma_{1}^{2} a_{1} \sin \epsilon, \\
\dot{\epsilon}_{1}=-k c_{1}^{1}+\gamma_{2}^{1} \frac{a_{2}}{a_{1}} \cos \epsilon, \quad \dot{\epsilon}_{2}=-k c_{2}^{2}+\gamma_{1}^{2} \frac{a_{1}}{a_{2}} \cos \epsilon .
\end{gathered}
$$

The superfix refers to the home-base at which any quantity is evaluated, the suffix labels each wave, and $\epsilon=\epsilon_{2}-\epsilon_{1}$ is their phase difference. $\left(c_{1}^{1}, c_{2}^{2}\right)$ are the Rossby wave speeds (3) at each home-base and

$$
\gamma_{j}^{i}=\left(\frac{v_{j}}{q_{i}} \frac{\partial \bar{q}}{\partial y}\right)^{i},
$$

for $i, j=1,2$, are the interaction coefficients. $\gamma_{2}^{1}$ describes the far-field influence of the meridional wind induced by wave- 2 at the home-base of wave- 1 (recall that the PV of wave- 2 is identically zero there).

In order to obtain growth of both waves, $(4 a, b)$ indicates that the interaction coefficients must have opposite signs. Assuming that the two home-bases are close 
enough to each other that $v_{1}^{1}$ and $v_{1}^{2}$ share the same sign and so do $v_{2}^{2}$ and $v_{2}^{1}$, then the signs of $\gamma_{2}^{1}$ and $\gamma_{1}^{2}$ are determined by the PV gradient at the two home-bases. Hereafter, we choose wave- 2 and wave- 1 to have positive and negative $\bar{q}_{y}$, respectively, at their home-bases. If the 'relative wind' at home-base $2, U^{2}-\left(U^{1}+U^{2}\right) / 2$, is positive then (3) indicates that each wave propagates at a rate $\gamma / k$ counter to the relative wind at its home-base. Hence, following Bretherton (1966b), we call these structures counterpropagating Rossby waves.

\section{(b) The orthogonality method using wave-activity conservation laws}

The 'orthogonality method' is based on the observation that the pseudomomentum and pseudoenergy of disturbances are globally conserved. In addition, the CRWs of the Eady model are orthogonal with respect to pseudomomentum and energy growth, whilst a pair of growing and decaying normal modes are, in general, not orthogonal to each other with respect to those norms (Held 1985). The parallel with the Eady CRWs is drawn upon by enforcing these two orthogonality conditions on the CRWs for general zonal flows. Pseudomomentum orthogonality makes transparent the Charney and Stern condition for instability, since then synchronous growth of both CRWs is possible only if the PV gradients weighted by CRW amplitude for the two CRWs take opposite signs. The second orthogonality property is related to the Fjørtoft condition for instability, since it implies that energy growth of both CRWs occurs only when the mean PV gradient and the zonal wind are positively correlated in the regions where the CRWs are concentrated. It also ensures that the CRWs represent positive and negative extrema of pseudomomentum given that the CRWs have equal amplitude under the energy growth norm discussed in Part I. There, it was shown that the CRWs which satisfy the above orthogonality relations also satisfy the CRW evolution equations $(4 a, b)$ and $(5 a, b)$, but the locally determined interaction coefficients (6) are replaced by

$$
\overline{\gamma_{j}^{i}}=\frac{\left\langle\eta_{i},-\bar{q}_{y} v_{j}\right\rangle}{\left\langle\eta_{i}, \bar{q}_{y} \eta_{i}\right\rangle}
$$

where $\eta_{i}=-q_{i} / \bar{q}_{y}$ is the meridional displacement of wave- $i$ and the inner product for wave-like quantities $p^{\prime}=\operatorname{Re}\left\{p_{k}(y, z) \mathrm{e}^{\mathrm{i} k x}\right\}$ is given by

$$
\left\langle p^{\prime}, q^{\prime}\right\rangle \equiv \iint \overline{p^{\prime} q^{\prime}} \bar{\rho} \mathrm{d} y \mathrm{~d} z=\iint \frac{1}{4}\left(p_{k}^{*} q_{k}+p_{k} q_{k}^{*}\right) \bar{\rho} \mathrm{d} y \mathrm{~d} z,
$$

where the overbar means zonal average and an asterisk indicates complex conjugate. The related Rossby phase speed of wave- $i$ is

$$
\overline{c_{i}^{i}}=\overline{U^{i}}-\overline{\gamma_{\mathrm{i}}^{i}} / k
$$

where

$$
\overline{U^{i}}=\frac{\left\langle\eta_{i}, U \bar{q}_{y} \eta_{i}\right\rangle}{\left\langle\eta_{i}, \bar{q}_{y} \eta_{\mathrm{i}}\right\rangle}
$$

can be described as the home-base wind against which CRW-i propagates.

It is important to note that the evolution of a CRW pair obtained by the orthogonality method can be described by the home-base CRW equations (3)-(6), provided that the following home-bases can be defined. If a point exists where the zero PV contour of wave-1, $q_{1}(y, z)=0$, intersects the zonal-wind contour $U(y, z)=\overline{U^{2}}$ in the meridional plane, this point can define the home-base of wave-2, $P_{2}$. Similarly, if $q_{2}(y, z)=0$ 
intersects $U(y, z)=\overline{U^{1}}$ this point can define $P_{1}$. The CRW phases, structures and phase speeds obtained by the home-base method for this particular $P_{1}$ and $P_{2}$ would be identical to those from the orthogonality method.

\section{(c) Phase-locking}

The equations for CRW evolution are simplified by renormalizing the CRW amplitudes $a_{1}$ and $a_{2}$ so that their interaction coefficients are equal and opposite:

$$
-\gamma_{2}^{1}=\gamma_{1}^{2}=\sigma
$$

reducing $(4 \mathrm{a}, \mathrm{b})$ and $(5 \mathrm{a}, \mathrm{b})$ to:

$$
\dot{\chi}=\sigma\left(1-\chi^{2}\right) \sin \epsilon, \quad \dot{\epsilon}=\mu+\sigma\left(\chi+\frac{1}{\chi}\right) \cos \epsilon,
$$

where $\chi=a_{1} / a_{2}$ is the amplitude ratio and $\mu=-k\left(c_{2}^{2}-c_{1}^{1}\right)$ would be the rate of change of phase difference in the absence of interaction. (12a,b) describes the evolution of any initial-value problem that can be formulated by normal-mode superposition, i.e. the discrete spectrum solution. The evolution is determined solely by the CRW initialamplitude ratio and phase difference, and by the CRW parameters $\sigma$ and $\mu$.

Normal modes are the particular solutions of $(4 a, b)$ and $(5 a, b)$ for which the two CRWs have equal growth rates and phase speeds. Equal growth rates imply equal amplitudes from $(4 \mathrm{a}, \mathrm{b})$ and $(11)$, whereas equal phase speeds $(\dot{\epsilon}=0)$ imply from $(12 \mathrm{~b})$ the phase locked angle

$$
\cos \epsilon_{+}=-\mu / 2 \sigma=\frac{k\left(c_{2}^{2}-c_{1}^{1}\right)}{2\left(-\gamma_{2}^{1} \gamma_{1}^{2}\right)^{1 / 2}} .
$$

The growth rate of the growing normal mode is

$$
\sigma_{+}=\sigma \sin \epsilon_{+},
$$

where $0<\epsilon_{+}<\pi$ and its phase speed is

$$
c_{\mathrm{r}}=-\dot{\epsilon}_{1} / k=-\dot{\epsilon}_{2} / k=\frac{1}{2}\left(c_{1}^{1}+c_{2}^{2}\right) .
$$

If the self-induced Rossby wave speed of the upper wave, $c_{2}^{2}$, exceeds that of the lower wave, $c_{1}^{1}$, (13) implies that $0<\epsilon_{+}<\pi / 2$, and so each wave 'helps' the other to propagate against the relative flow at its home-base. If, on the other hand, the upper wave moves more slowly, then $\pi / 2<\epsilon_{+}<\pi$ and, thus, each wave 'hinders' the counterpropagation of the other, i.e. each wave acts to move the other in the direction of the relative flow at its home-base.

\section{THE CHARNEY MODEL AND ITS DiSPERSION RELATION}

The basic state of the Charney model is a zonal flow with a constant positive shear, $\Lambda$, and stratification on a $\beta$ plane with a lower boundary at $z=0$ :

$$
U=\Lambda z \quad \text { for } 0 \leqslant z<\infty, \quad N=\text { const }, \quad f=f_{0}+\beta y,
$$

where $N$ is the Brunt-Väisälä frequency and $f_{0}$ is the domain-averaged Coriolis parameter. For simplicity, we consider the case, examined by Kuo (1973), in which the density is constant and disturbances are independent of $y$. The basic-state PV gradient 
is then

$$
\bar{q}_{y}=\beta-\left(\frac{f_{0}}{N}\right)^{2} \Lambda \delta\left(z-0^{+}\right),
$$

where the Bretherton (1966a) $\delta$-function representation of boundary temperature has been used. The normal-mode solution for PV can be written as

$$
q^{\prime}=Q_{k}(z) \mathrm{e}^{\mathrm{i} k(x-c t)} .
$$

The structure $Q_{k}$ includes the Bretherton $\delta$-function representation,

$$
Q_{k}(z)=Q_{\text {int }}(z)+\left(\frac{f_{0}}{N^{2}}\right) \Theta \delta\left(z-0^{+}\right),
$$

where $Q_{\text {int }}$ represents the interior PV and $\Theta$ is the buoyancy $\left(\Theta=g \theta^{\prime} / \theta_{0}=f_{0} \psi_{z}\right)$. Using the Fourier decomposition ( $2 \mathrm{a}, \mathrm{b})$, the quasi-geostrophic interior PV structure can be expressed in terms of the meridional wind as

$$
Q_{\text {int }}=k\left\{v_{k}-\frac{1}{k^{2}}\left(\frac{f_{0}}{N}\right)^{2} \frac{\partial^{2} v_{k}}{\partial z^{2}}\right\},
$$

where, from here on, the suffix $k$ will be dropped. Since positive surface meridional wind advects both warm surface potential temperature from the south and a negative anomaly of planetary PV, (1) yields that, just above the surface,

$$
\Theta=-\left(\frac{f_{0} \Lambda}{\beta}\right) Q_{\text {int }} \quad \text { at } z=0^{+} .
$$

The solution of (1), using (16)-(21), is obtained in terms of the confluent hypergeometric functions (see e.g. Pedlosky 1987). The vertical scale can be normalized by $H=\left(f_{0} / N\right)^{2} \Lambda / \beta$, which indicates the depth over which the interior PV gradient must be integrated to equal the boundary contribution in (17). Other appropriate scalings can be obtained from the Charney-model parameters: a horizontal scale $L=\left(N / f_{0}\right) H$, a velocity scale $U_{0}=\Lambda H$, and a time scale $T=L / U_{0}$. Using the following typical mid-latitude values: $f_{0}=10^{-4} \mathrm{~s}^{-1}, \beta=1.6 \times 10^{-11} \mathrm{~m}^{-1} \mathrm{~s}^{-1}, N=1.1 \times 10^{-2} \mathrm{~s}^{-1}$ and $\Lambda=3 \times 10^{-3} \mathrm{~s}^{-1}$ one finds that $H \approx 15 \mathrm{~km}, L \approx 1650 \mathrm{~km}, U_{0} \approx 45 \mathrm{~m} \mathrm{~s}^{-1}$ and $T \approx 10 \mathrm{~h}$.

The normalized normal-mode dispersion relation as a function of $r=1 / 2 k=$ $\{1 /(4 \pi L)\} \times$ dimensional wavelength, is shown in Fig. 1(a). Since $r=1$ corresponds to a wavelength of about $21000 \mathrm{~km}$, the synoptically relevant modes, which were found by Charney (1947), lie within $0<r<1$ and have both the growth rate and phase speed zero at either end of this range, while the most unstable mode occurs at $r \approx 0.63$. For larger wavelengths, less rapidly growing modes have been found by Green (1960) and Burger (1962) with different branches in each range $n<r<n+1$, where $n$ is an integer. The maximal growth rate of each branch decays with $n$. Hereafter, the range $n<r<n+1$ is referred to as the Green- $n$ branch.

Besides the decaying modes, which are the complex conjugates of the growing modes, Miles (1964) showed the existence of branches of neutral modes with negative phase velocity. From each neutral point $(r=n)$ a branch of neutral modes appears with a westward phase speed that increases with wavelength. The first two neutral branches are indicated in Fig. 1(b). A similar diagram was obtained by Geisler and Dickinson (1975) for the more general case where the surface wind is greater than zero $\left(U=U_{\mathrm{s}}+\Lambda z\right)$. 

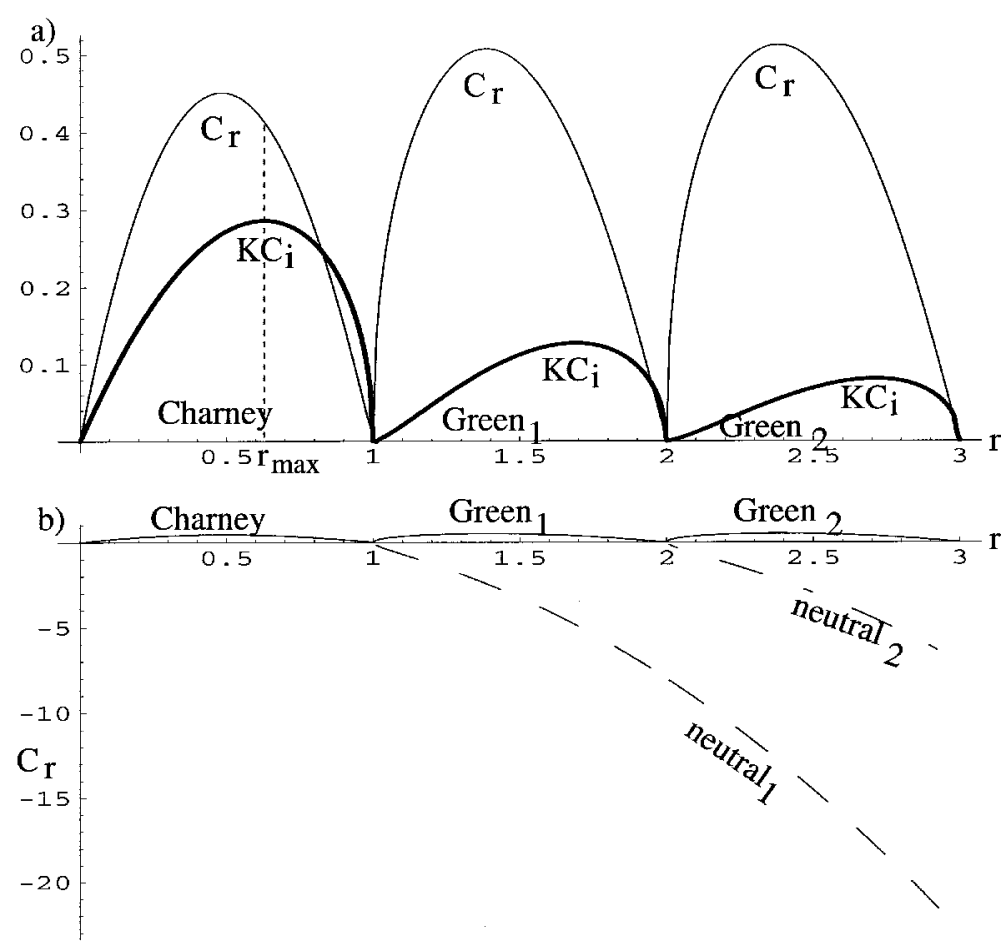

Figure 1. The dispersion relation for the normal modes in the Charney model. (a) The phase speed of the unstable modes $c_{\mathrm{r}}$ (scaled by $U_{0}$ ) and their growth rate $k c_{\mathrm{i}}$ (scaled by $T^{-1}$ ) are indicated by the solid and thick lines, respectively, as a function of the non-dimensional wavelength $r$. Neutral points occur at integer $r$ separating the Charney branch $(0<r<1)$ from the Green- $n$ branches $(n<r<n+1)$. (b) The phase speed $c_{\mathrm{r}}$ of the most unstable branches and the first two branches of neutral modes (note the change in scale from (a)).

The 'triple points' at the junctions between two unstable branches and a neutral branch have steering levels below ground in this case and, where $c_{\mathrm{r}}(r) \leqslant U_{\mathrm{s}}$, all modes are neutral.

The most unstable Charney modes are shifted to shorter more realistic wavelengths if the density falls exponentially with height scale $H_{\rho}$, as in the original study of Charney (1947). Following Lindzen et al. (1980), the condition for neutral points (Burger 1962; Miles 1964) can be expressed in terms of wave number (here scaled by $N H / f_{0}$ rather than $\left.N H_{\rho} / f_{0}\right)$ as

$$
k^{2}=\left(\frac{1+H / H_{\rho}}{2 n}\right)^{2}-\left(\frac{H / H_{\rho}}{2}\right)^{2} .
$$

The first neutral point $(n=1)$ corresponds to wavelength $r=1 / 2 k=(1+2 H /$ $\left.H_{\rho}\right)^{-1 / 2}$ which is 0.45 for a realistic $H_{\rho}=7.5 \mathrm{~km}$, compared with unity for the constantdensity case $H / H_{\rho}=0$. Also, all the higher neutral points cease to exist for $H / H_{\rho}>1$. The dispersion curves are also sensitive to the wind profile. Geisler and Garcia (1977) and Lindzen et al. (1980) have shown that, when $U(z)$ reaches a maximum in the upper troposphere, the first neutral point is destabilized (there is still a local minimum in $c_{\mathrm{r}}$ and $c_{\mathrm{i}}$ but they are both positive) and moves to shorter wavelengths and all higher neutral points are completely eliminated.

Despite the sensitivity of the solutions to unrealistic properties of the basic state (especially linear shear), the constant-density Charney model represents the simplest one 
for which solutions have been examined in detail. In the following section we analyse this solution from the CRW perspective starting, as an example, with the most unstable mode at $r \approx 0.63$.

\section{THE CRW ANALYSIS FOR THE CHARNEY MODEL}

\section{(a) The most unstable mode}

Cross-sections through the most unstable normal mode and its decaying complex conjugate are shown in Figs. 2(a) and (b), where the vertical and the zonal scales are normalized by $H$ and the wavelength, respectively. The growing mode interior PV structure, indicated by the heavy solid contour lines, tilts eastward with height in contrast to the meridional wind structure, indicated by the solid contour lines, which tilts westward with height. The steering level of this mode (where $U(z)=c_{\mathrm{r}}$ ) is located at approximately $0.43 \mathrm{H}$.

Figures 2(e) and (f) show the upper and lower CRWs obtained using the orthogonality method. These CRWs possess the maximum and minimum pseudomomentum, respectively, that it is possible to achieve by combining the fastest-growing normal mode and its complex conjugate, subject to the constraint that they contribute equally to energy growth. For this reason, the lower CRW has its greatest PV amplitude at the ground where the boundary temperature gradient can contribute to negative pseudomomentum. The upper CRW has its greatest PV magnitude away from the ground and, therefore, has positive pseudomomentum. It is clear that the CRWs have wave activity focused at two distinct levels.

However, in order to apply Bretherton's (1966b) two-level perspective on baroclinic growth exactly, it is necessary to use the home-base method to determine the CRWs. Since the PV gradient is only negative at the ground, it is a natural choice to locate the home-base of the lower CRW there. This is sufficient to determine the structure of the upper CRW by choosing the combination of growing and decaying mode that gives zero PV signature at the surface. Figure 2(c) shows the structure of this upper CRW which contains a distinct PV maximum at the level of approximately $0.7 \mathrm{H}$ (indicated by the dashed horizontal line in Figs. 2(a), (c) and (d)). Note its similarity to the upper CRW obtained by the orthogonality method (Fig. 2(e)) and the structure of meridional PV flux induced by surface temperature shown in Fig. 3 of Robinson (1989).

A reasonable choice is to locate the home-base of the upper CRW at this maximum which, in turn, determines the structure of the lower CRW as the normal-mode combination with zero PV there (shown in Fig. 2(d)). Again, note its similarity to the lower CRW obtained by the orthogonality method (Fig. 2(f)) and to the structure of PV flux induced by PV at the home-base of the upper CRW, as shown in Fig. 4 of Robinson (1989). Although the CRWs have continuous PV structure, Robinson's analysis highlights how the PV fluxes induced by just PV anomalies at the two home-bases resemble the CRW structures themselves. This suggests that the normal-mode growth can be described by the interaction of two CRWs, characterized by their PV anomalies at these home-bases.

The basic discussion of CRW interaction at two levels can now be applied. The home-base CRW theory (section 2(a)) quantifies all features of this interaction, given the normal-mode structure. The two CRWs are untilted with meridional winds that are in quadrature with the PV, so that each wave by itself can propagate but cannot grow. For the upper wave, the negative meridional wind, indicated by $-v$, lies onequarter wavelength to the west of the positive PV anomaly, indicated by $+q$, and therefore advection of large planetary PV from the north tends to shift the positive PV anomaly to the west, causing the upper wave to propagate westward. For the lower 

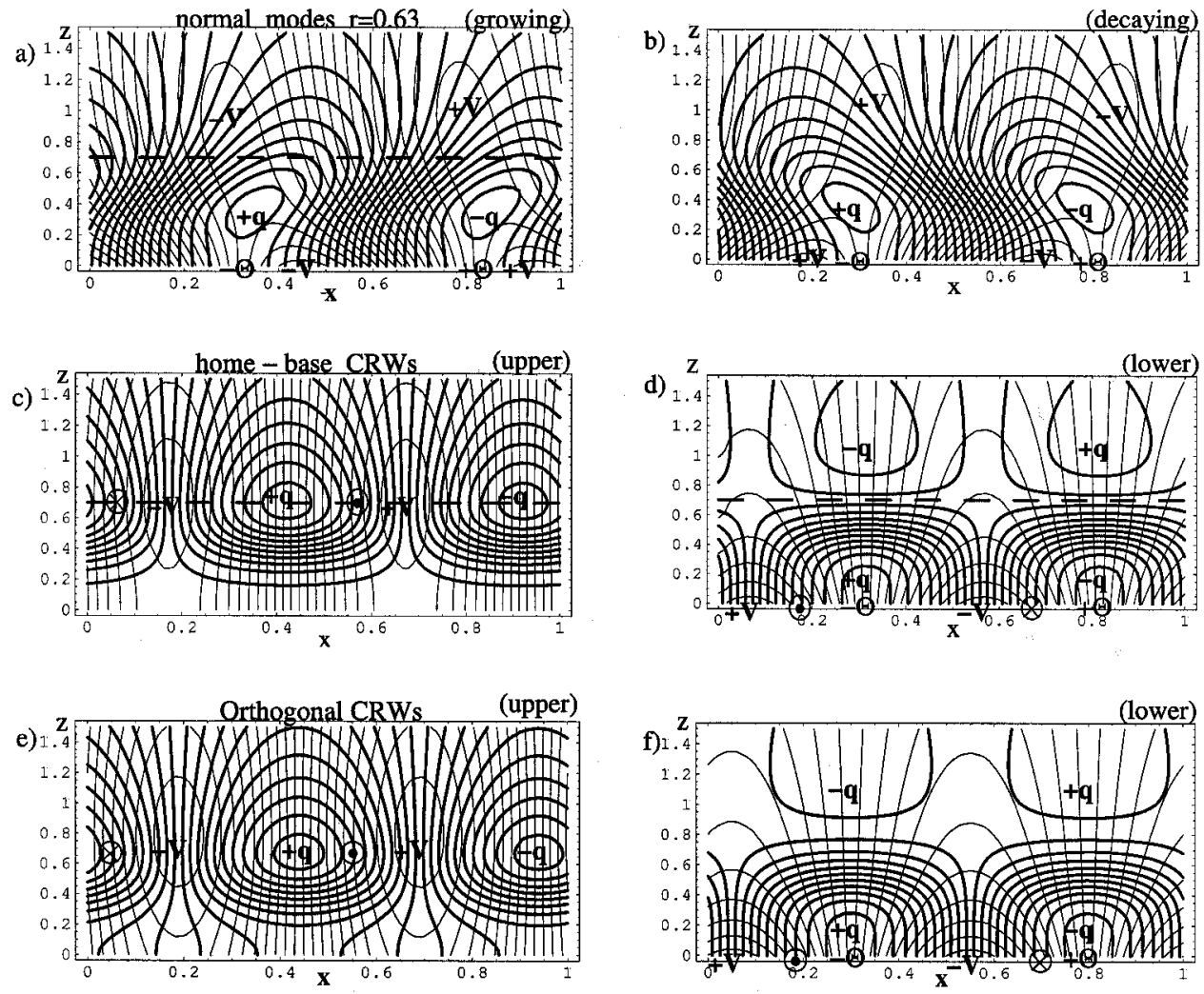

Figure 2. Cross-sections showing (a) the fastest-growing normal mode, (b) its decaying complex conjugate, (c) and (d) the upper and lower CRWs obtained by the home-base method, (e) and (f) the upper and lower CRWs obtained by the orthogonality method. The perturbation PV, $q$, is shown by thick contours and the meridional wind, $v$, by thin contours. The surface potential-temperature anomalies are indicated by $\Theta$. The horizontal dashed lines in (a),(c) and (d) represent the upper CRW's home-base. The positive and negative meridional wind that each CRW induces on the other CRW's home-base is indicated by $\bigotimes$ and $\odot$, respectively.

wave, the negative meridional wind at the surface lies one-quarter wavelength to the east of the negative potential temperature anomaly, indicated by $-\Theta$, which lies under a positive interior PV anomaly. Advection from the north brings cold temperature and high planetary PV, tending to shift the negative temperature anomaly, and the positive interior PV just above it, to the east, causing the lower wave to propagate eastward.

The upper and lower CRWs are shown with a phase and amplitude ratio such that their sum gives the growing normal mode (Fig. 2(a)). Although the interior PV of the growing mode tilts eastward with height, when decomposed onto the two CRWs we can see that the positive PV anomalies of the upper wave lie to the west of the positive surface-temperature anomalies. The cyclonic circulation associated with positive temperature anomalies dominates the anticyclonic circulation induced by the lower CRW's interior PV, so that the phase of the lower CRW is defined by the phase of the surface temperature wave. The normal-mode phase difference $\epsilon_{+}=\epsilon_{2}-\epsilon_{1} \approx 0.79 \pi$ (cf. $\epsilon_{+} \approx 0.77 \pi$ for the most unstable Eady mode), and thus the CRWs make each other grow but also hinder the counter-propagation of the other (see Fig. 2 of Part I). Hence, on the home-bases, the CRW configuration matches the general picture shown in Fig. 1(b) of Part I. 
Note that, although the growing normal mode has a PV amplitude maximum close to the steering level $(0.43 \mathrm{H}$ in Fig. 2(a)), the upper CRW is concentrated considerably higher than this and is a maximum at $0.7 \mathrm{H}$. The dimensional height of $10.5 \mathrm{~km}$ is exaggerated by the assumption of constant density. However, even without a tropopause in the basic state, baroclinic instability is seen to involve the interaction of a lower CRW dominated by a surface temperature wave and an upper CRW at mid- to uppertropospheric levels.

Despite the similarities in the dynamics of their fastest-growing modes, there are significant differences in the dispersion relations for the normal modes of the Charney (1947) and the Eady (1949) models. The Eady model has a short-wave cut-off not observed in the Charney model. This arises because the counter-propagation of Eady edge waves becomes too weak to maintain phase-locking against the shear when their depth scale, which is proportional to horizontal wavelength, becomes too small relative to the specified separation of the boundaries. However, in the Charney model the upper CRW can always interact as wavelength decreases by existing closer to the lower boundary on the interior PV gradient.

More difficult to understand are the neutral points of integer $r$ (see Fig. 1) which separate branches of growing normal modes in the Charney model. No analogous behaviour occurs in the Eady model. Each neutral point forms a triple junction between two branches of growing (and decaying) modes and one branch of neutral modes. Motivated by this difference, in the next section we suggest an explanation for the normal-mode behaviour in the vicinity of the neutral points, from the CRW perspective.

\section{(b) The neutral points and neutral branches}

At the first neutral point, $r=1 / 2 k=1$, the normalized PV and meridional wind structures of the normal mode are given (e.g. Pedlosky 1987) by:

$$
Q_{\text {int }}(z)=-\Theta \mathrm{e}^{-k z}, \quad v(z)=-\Theta k z \mathrm{e}^{-k z} .
$$

Normalizing by $\Theta$, the interior PV as a function of height is indicated by the thick line in Fig. 3(a) and the meridional wind is indicated by the thick lines in Figs. 3(b) and (c). Assuming that $\Theta$ is positive, the interior PV is negative everywhere. The meridional wind in the interior has the same sign as the PV and, therefore, according to (2) the flow circulates anticyclonically around the negative interior PV anomaly, as expected from a simple PV structure. The mode is stationary since the Rossby counter-propagation rate, $-\gamma / k$, is negative and balances the mean wind $U$ exactly at every level.

Although the surface temperature gradient is negative, the surface temperature anomalies do not propagate to the east because the meridional wind is zero at the surface. This results from an exact cancellation of $v(0)$ induced by positive surface potentialtemperature anomalies and the integrated effect of the opposite signed PV anomalies above. Following Robinson's (1989) method and using the Green function suggested by Davies and Bishop (1994) and Bishop and Heifetz (2000), it is shown in the appendix that the surface velocity induced by the interior PV of (23a) is $v_{q}(0)=-\Theta r$, whereas the velocity induced by the surface-temperature anomaly is simply $v_{\theta}(0)=\Theta$ and, therefore, at $r=1$ the cancellation of the surface meridional wind is exact. Moreover, in the interior, we can use (A.1) and (A.2) to verify that the negative contribution $v_{q}(z)$ outweighs the positive contribution $v_{\theta}(z)$ everywhere. $v_{\theta}(z), v_{q}(z)$ and their sum $v(z)$ are indicated in Fig. 3(c) by the solid, dashed and thick lines, respectively.

The steering level of the mode at $r=1$ is located on the ground. However, if we slightly increase $r$ then we expect $v_{q}(0)$ to be more negative than $-\Theta$, while 

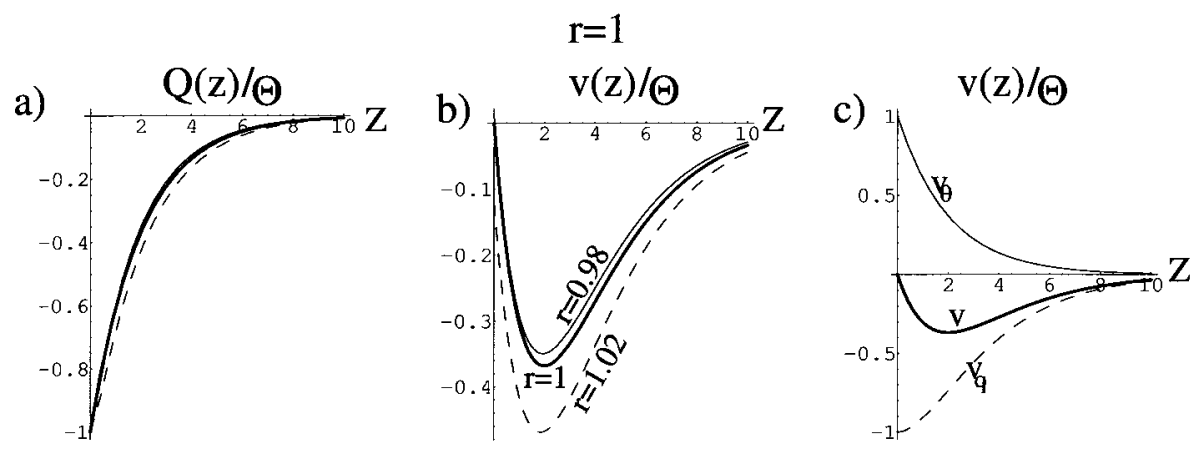

$\mathrm{r}=2$
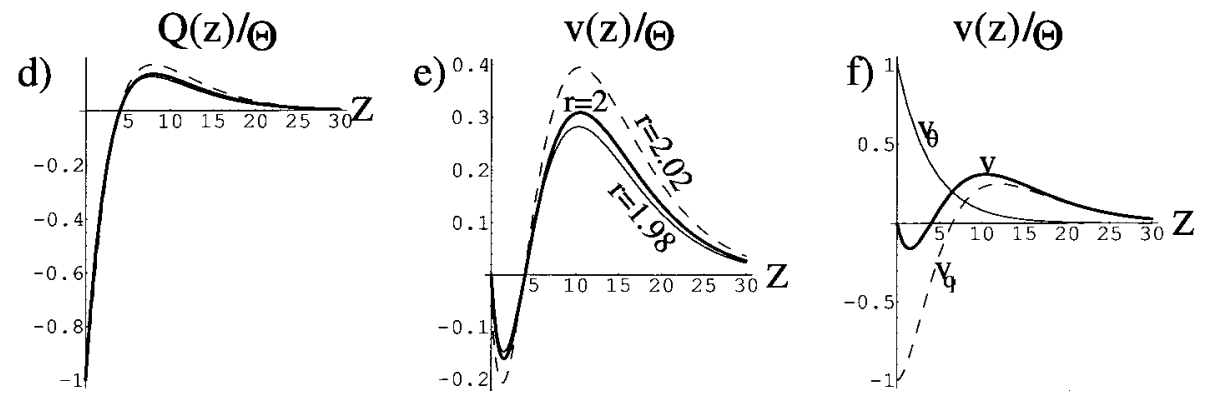

Figure 3. The vertical structure of the normal modes in the vicinity of the first two neutral points. (a) The interior PV, $Q$, scaled by surface temperature, $\Theta$, for the neutral modes $r=1$ (thick line), $r=1.02$ (dashed line) and the amplitude of the growing mode $r=0.98$ (thin line). (b) The associated meridional wind, scaled by $\Theta$, for the same modes. (c) The meridional wind for the $r=1$ mode decomposed into the contributions induced by surface temperature, $v_{\theta}$, and interior PV, $v_{q}$, calculated using (22) and the appendix. Panels (d), (e) and (f) are the same as (a), (b) and (c), but in the vicinity of the second neutral point $r=2$.

$v_{\theta}(0)$ is unchanged. Consequently, we expect $v(0)$ to become slightly negative so that positive surface $\theta$ anomalies are circulated by anticyclonic motion resulting in westward propagation and, hence, a steering level which lies 'underground'-external to the flow. In Figs. 3(a) and (b) the profiles of PV and $v$ for the neutral mode at $r=1.02$, are shown by dashed lines. As expected, the surface meridional wind is indeed negative and small. Owing to the nature of the inversion of (20), amplification of the interior PV yields an even a larger amplification in $v$, so that $v / q$ increases. Hence, the counter propagation rate toward the west, $\gamma / k$, exceeds the eastward wind, $U$, at all levels and the mode is able to propagate coherently to the west. For larger wavelengths, we therefore expect the propagation rate toward the west to increase, as seen in Fig. 1(b).

If the wavelength is slightly less than one, then $v(0)$ induced by surface-temperature anomalies dominates the interior PV influence, and consequently the propagation rate, $-\gamma / k$, on the surface becomes positive. Hence, any coherent mode with such a surface signature would be expected to have a steering level located just above ground where the phase speed can match the zonal flow. Bretherton (1966a) showed that modes with steering levels above ground must be unstable $\left(c_{\mathrm{i}} \neq 0\right)$ or have zero amplitude there. Since the mean PV gradient, $\beta$, is constant everywhere in the interior, $v$ would also have to vanish at the steering level in order for $\gamma$ to be zero, so that $c=U$ in (3). Since extrapolation of (23b) for $r<1$ does not imply vanishing $v$ in the interior, the 
solution cannot be described by a single, untilted Rossby wave of this form. It is shown in section 2(c) that two CRWs are required to describe the unstable branches.

However, the PV and $v$ profiles of the growing Charney mode at $r=0.98$ are indicated by the solid lines in Figs. 3(a) and (b). Since these structures are only slightly tilted with height, only their magnitudes are shown here. As expected, the surface meridional wind is indeed very small, but positive. The strength of the interior PV becomes slightly smaller, making the interior meridional wind less negative. Consequently, the westward propagation rate becomes slightly smaller than the eastward mean wind $U$, at all heights, resulting in an eastward phase velocity.

At the second neutral point, $r=2$, the normal mode PV and $v$ structures,

$$
Q_{\text {int }}(z)=-\Theta(1-k z) \mathrm{e}^{-k z}, \quad v(z)=-\Theta(1-k z) k z \mathrm{e}^{-k z},
$$

are indicated, respectively, by the thick lines of Figs. 3(d) and (e). The structure of the mode is similar to the one at the first neutral point in the sense that interior PV and the meridional wind have the same sign everywhere. Both change sign at $z=2 r=4$. The induced wind $v(z)$ and its components $v_{\theta}(z)$ and $v_{q}(z)$, are indicated in Fig. 3(f) by the heavy, light and dashed lines, respectively. Although the wavelength is twice as large as at the first neutral point, the steering level remains on the ground because the upper PV chunk $(z>4)$ induces a meridional wind of the same sign as the surface temperature, and together they balance exactly $v$ induced by the lower PV chunk $(0<z<4)$. The surface meridional wind induced by the surface temperature remains $v_{\theta}(0)=\Theta$. However, the integrated effect of the interior PV is $v_{q}(0)=-\Theta r / 2$ (using (A.3)) which can be divided into $-\Theta(r / 2)\left(1+\mathrm{e}^{-r}\right)$ and $\Theta(r / 2) \mathrm{e}^{-r}$ contributions from the lower and upper PV chunks, respectively.

Extrapolating (24) to $r$ slightly larger than 2 yields a neutral Rossby wave with an 'underground steering level', while extrapolating for $r$ slightly smaller than 2 yields a steering level above ground which implies the existence of two CRWs. Therefore, similar to the first neutral point, the second point forms a junction between a branch of neutral modes to its right (the dash-dot line in Fig. 1(b)) and the Green-1 branch of unstable modes to its left (solid line between $1<r<2$ in Figs. 1(a) and (b)). Geisler and Dickinson (1975) also noted that, for the neutral branches, the PV gradient $\beta$ 'drives the mode towards the west' overcoming the 'opposite effect driving the mode toward the east' associated with the surface temperature gradient. They also observed the increase in number of vertical nodes on the higher neutral branches, although they did not discuss the scale effect of PV inversion and its role in changing the Rossby wave speeds.

Similar behaviour is found at the neutral points $r=n$ for higher integer $n$. The number of sign changes in the interior $\mathrm{PV}$ and $v$ structure is equal to $n-1$. Additionally, the vertical penetration of the structures increases greatly with $n$ (note the change in scales between Figs. 3(a) and (d)). If the structure remains essentially the same as at $r=n$, then for $r>n$ interior PV dominates surface temperature in the inversion to obtain velocity, resulting in the neutral- $n$ branches describing Rossby waves whose westward phase speed increases indefinitely with $r$. However, by changing the vertical structure at the next neutral point, $r=n+1$, the opposite signed PV chunks cancel each other in the induction of surface wind to such an extent that their integrated effect exactly cancels the wind induced by the surface temperature wave. The result is a new stationary neutral mode.

So far, it has been explained how neutral points result from an exact cancellation of the integrated effects of interior PV structure with the effects of the surface temperature wave. The existence of the neutral branches for $r>1$ develops naturally from considering the different scale-dependence of inversion for surface temperature and interior PV. 
a)

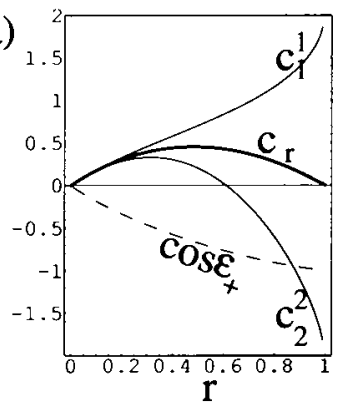

d)

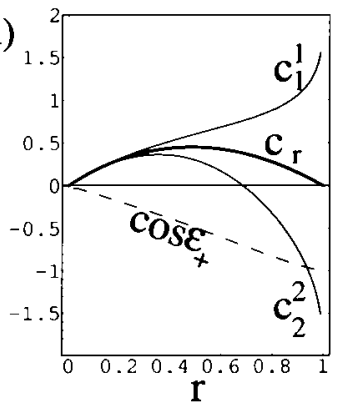

b)

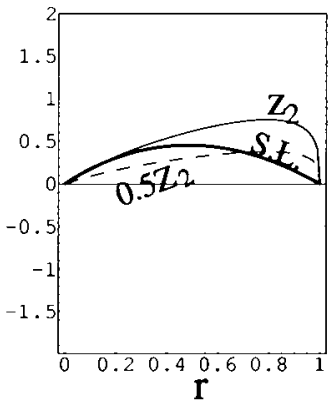

e)

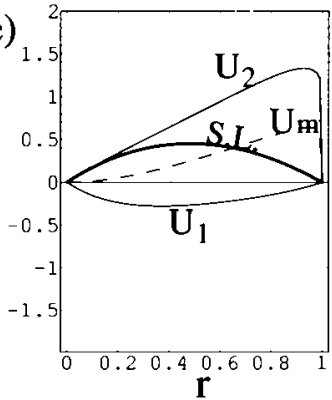

c)

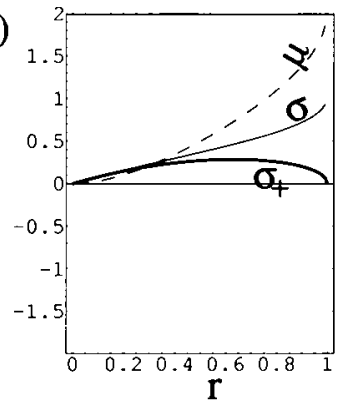

f)

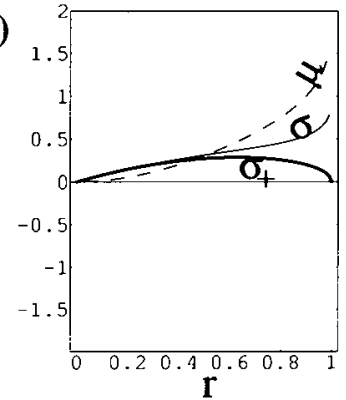

Figure 4. The properties of the Charney branch $(0<r<1)$ normal modes and associated CRWs, as functions of scaled wavelength $r$. (a) The lower and upper CRWs' phase speeds, $c_{1}^{1}$ and $c_{2}^{2}$ (thin lines), and their average which equals the normal mode phase speed $c_{\mathrm{r}}$ (thick line). The phase difference between the CRWs when phase-locked into the growing mode equals $\epsilon_{+}$(dashed line). (b) The location of the upper CRW's home-base, $z_{2}$ (scaled by $H$ ) shown relative to the steering level, which equals $c_{\mathrm{r}}$ under the normalization (thick line). (c) The fundamental parameters for the CRW behaviour indicating the rate of phase change due to the difference in the Rossby wave speeds (without interaction), $\mu$, and their normalized interaction coefficient, $\sigma$. The normal-mode growth rate is $\sigma_{+}=k c_{\mathrm{i}}$ (thick line). Panels (d), (e) and (f) are similar to (a), (b) and (c), but the CRWs were obtained by the orthogonality method rather than the home-base method. In (e) the upper and lower CRWs' home-base winds $\bar{U}_{2}$

and $\bar{U}_{1}$, calculated from (10) and scaled by $U_{0}$, are shown (thin lines) with their average $\bar{U}_{\mathrm{m}}$ (dashed lines).

In the following sections, similar arguments are used to explain features of the unstable Charney and Green branches, but in these cases the interior PV is partitioned between two CRWs, the lower of which contains the surface temperature wave.

\section{(c) The Charney branch}

(i) CRW behaviour calculated from normal modes. As demonstrated in section 4(a), the growing (or decaying) normal modes can be alternatively described by two phaselocked CRWs. In this section, the changes in CRW propagation rates, strength of interaction and structure as $r$ is varied across the Charney range $(0<r<1)$ are used to understand the growing normal-mode structures and their dispersion relation.

Figure 4 shows the properties of the Charney-branch normal modes and CRWs as functions of wavelength $r$. The CRWs have been obtained by the home-base method in Figs. 4(a), (b) and (c) and by the orthogonality method in Figs. 4(d), (e) and (f). For all wavelengths, the lower-wave phase speed, $c_{1}^{1}$, is larger than the upper-wave phase speed, $c_{2}^{2}$ and, therefore, in order to remain phase-locked, each CRW must hinder the counter-propagation of the other (see (3) and (6) for the Rossby wave-speed definition). Therefore, physical CRW arguments (i.e. Fig. 1 in Part I) indicate that the phase difference $\epsilon_{+}$must lie between $\pi / 2$ and $\pi$ or, equivalently, $0>\cos \epsilon_{+}>-1$. Equation (13) quantifies this relationship. 
The height, $z_{2}$, of the upper CRW's home-base (the height of its PV maximum) is shown in Fig. 4(b), together with the height of the steering level (equal to the normalized $c_{\mathrm{r}}$ in Fig. 4(a)). The upper wave's home-base always lies above the steering level. However, as $r \rightarrow 0$ the upper CRW is located almost on the steering level and both intrinsic Rossby wave speeds approach the normal-mode phase speed. Consequently there is little need for hindering and the normal-mode growth rate, $\sigma_{+}$, approximately equals the maximal growth, $\sigma$, since $\epsilon_{+} \approx \pi / 2$.

The difference in the self-induced wave speeds of the CRWs, $\mu$, clearly increases rapidly with $r$ (Figs. 4(c) and (f)) indicating that increasing interaction is necessary to hinder each CRW's propagation sufficiently for phase-locking to occur. The normalized interaction coefficient, $\sigma$, does indeed increase with $r$. However, the normal-mode growth rate, $\sigma_{+}=k c_{\mathrm{i}}$, peaks at $r \approx 0.63$ and reduces to zero as $r \rightarrow 1$. This occurs because the CRWs approach a maximally hindering configuration where they are $\pi$ out of phase, and thus no growth through interaction is possible, as can be argued physically from Fig. 1 of Part I.

The home-base winds, $\bar{U}_{1}$ and $\bar{U}_{2}$, obtained from the orthogonality method (10), and their average $\bar{U}_{\mathrm{m}}$ are shown in Fig. 4(e). Although, $\bar{U}_{1}$ is external to the flow for all wavelengths*, the reference wind $\bar{U}_{\mathrm{m}}$ has similar behaviour to the mid point between the upper wave's PV maximum, obtained by the home-base method, and the ground (labelled 0.5 $z_{2}$ in Fig. 4(b)).

The similarity of the CRWs obtained from the normal modes by the home-base and orthogonality methods has a number of consequences. The orthogonality method is objective and is based on the constraints that the CRWs should have to contribute equally to pseudomomentum (but with opposite signs) and to energy growth, and that they should be orthogonal with respect to these global properties. These are important features of CRWs obtained independently of the normal modes for the Eady and two-layer models. The fact that the home-base CRWs are so similar implies that the subjective choices made for home-base locations also retain these physical properties approximately. Furthermore, it can be assumed that the physical intuition gained from the PV-thinking perspective of CRW interaction (i.e. Fig. 1 of Part I), which motivates the home-base method, applies with good approximation to the orthogonal CRWs.

(ii) CRW structures at the limits of the Charney range. In the short-wavelength limit, the upper CRW's PV structure collapses towards a $\delta$-function, as illustrated for $r=0.1$ and $r=0.02$ in Fig. 5(a). The lower CRW's interior PV structure (Fig. 5(b)) develops extrema for $r=0.1$ and as $r$ decreases further; these resemble a positive and negative spike just above and below $z_{2}$ respectively which cancel in the inversion to obtain velocity. Assuming that the lower CRW is dominated by the surface temperature wave, then we can use (3), (6), (17), (19) and (A.3) to show that its Rossby wave speed $c_{1}^{1}=-\gamma_{1}^{1} / k \approx 2 r$, as is the case for the semi-infinite Eady edge wave when the surface zonal wind is zero. Actually, $c_{1}^{1}<2 r$ since the lower CRW includes an interior PV anomaly below $z_{2}$, with opposite sign to the surface temperature, reducing the meridional wind induced at the surface.

While the surface edge wave propagates at speed $2 r$, an interior PV $\delta$-function cannot counter-propagate (since $v_{2}$, from (A.1), is finite, but $\gamma_{2}^{2}=\left(v_{2} \beta / q_{2}\right)^{2}$ is zero at the $\delta$-function) and, thus, $c_{2}^{2}$ is equal to the flow at its home-base (from (3)).

\footnotetext{
* The fact that the home-base wind $\bar{U}_{1}$ is negative could be interpreted as if the 'centre' of the orthogonal lower CRW is located underground. This can be rationalized by noting that the surface PV $\delta$-function can be replaced by an equivalent PV distribution that fills the region below ground.
} 

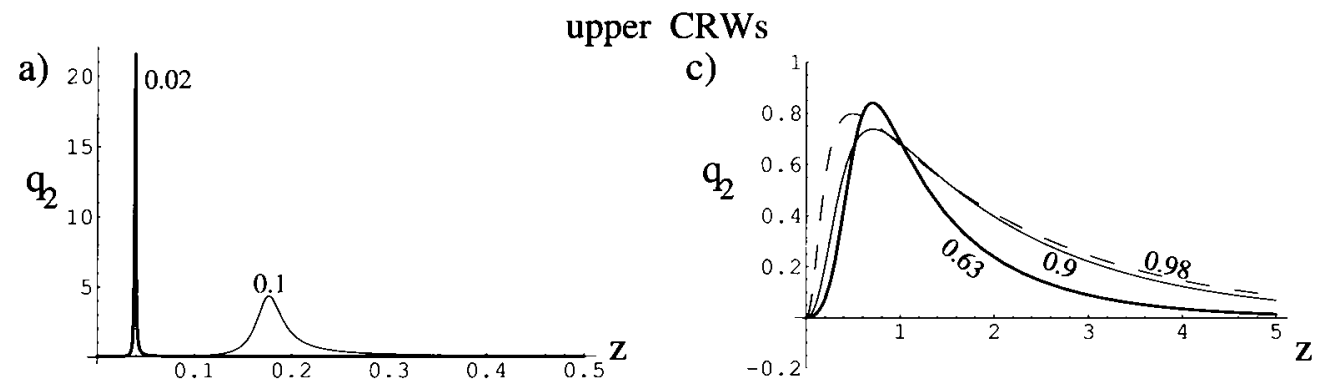

lower CRWs
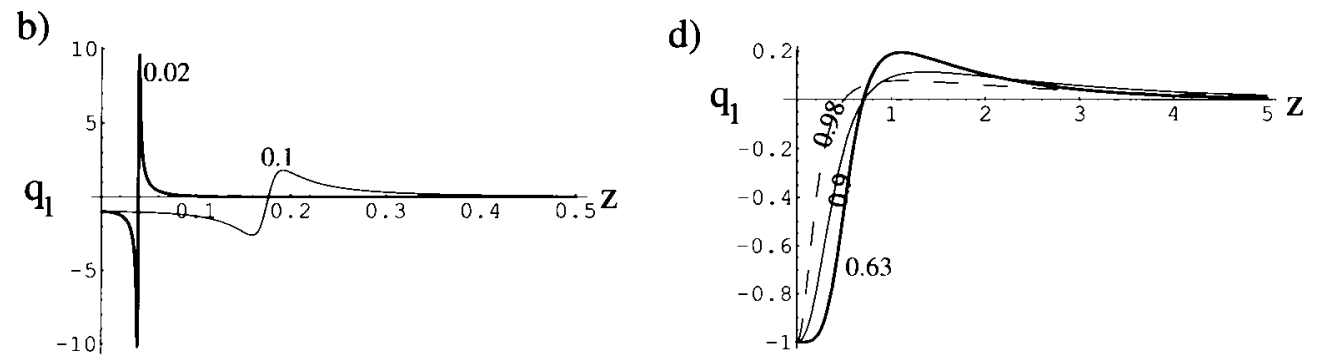

Figure 5. The vertical structures of the upper and lower CRWs from the Charney branch, as constructed by the home-base method. Their amplitudes are chosen so that, when superposed with the correct phase difference $\epsilon_{+}$, they reconstruct the growing normal mode normalized by its surface temperature $\Theta$. The upper and lower CRWs for $r=0.02$ and 0.1 are indicated in (a) and (b) by the thick and thin lines, respectively. The profiles for $r=0.63$, 0.9 and 0.98 are indicated in (c) and (d) by the thick, thin and dashed lines, respectively.

Since interaction is also weak (from (6)), phase-locking only occurs when $c_{2}^{2} \approx c_{1}^{1}$ (from (13)) and, thus, the flow at $z_{2}$ approximately matches the lower CRW speed. Normalmode phase speed is the average of the Rossby wave speeds and, therefore, as $r \rightarrow 0$ the upper CRW coincides with the steering level. This situation was described as criticallayer instability by Bretherton (1966a) and can be viewed exactly as the interaction of two independent CRWs (as discussed by Bretherton (1966b) for the two-layer model). Interestingly, it is the limit in which overreflection estimates (Lindzen et al. 1980) are accurate since $c_{\mathrm{i}} \rightarrow 0$. Zurita and Lindzen (2001) have also noted that Charney short waves (where $k^{-1} f_{0} / N<H$ or, equivalently, $r<0.5$ ) are dominated by the surface temperature wave and have slightly slower phase speeds than a pure edge wave, due to the modification by interior PV anomalies.

At the opposite end of the Charney range, $r \rightarrow 1$, it has already been shown (Fig. 3(a)) that the normal-mode interior PV structure approaches the exponential shape (23a) of the neutral mode which has a maximum at the ground. Figure 5(c) shows how the vertical penetration of the upper CRW increases with $r$, even though its homebase location must collapse towards the ground as $r \rightarrow 1$. The peak in PV is seen to decrease in height for $r>0.9\left(z_{2}\right.$ in Fig. 4(b)). The level of zero PV for the lower CRW (Fig. 5(d)) follows the upper CRW maximum by definition. At $r=0.98$ the two CRWs are virtually in anti-phase $\left(\epsilon_{+} \approx \pi\right)$ and the PV of the growing normal mode (Fig. 3(a)) can be obtained approximately by subtracting the upper CRW from the lower (Fig. 5(d)-(c)). As the neutral point $r=1$ is approached, the lower CRW becomes a pure surface temperature wave and the upper CRW contains all of the interior PV. 
This is precisely the situation discussed for the neutral mode in section 4(b). Thus, from the CRW perspective, there is a smooth transition across the neutral point from the CRW structures of the unstable Charney branch into the structure of the neutral1 modes. For both branches, the behaviour of the normal modes can be described to a good approximation by the interaction of a surface temperature wave with an upper CRW containing most of the interior PV.

(iii) Normal-mode behaviour deduced by assuming simple CRW structures. The exact solutions for the Charney basic state are known. However, analytic solutions for slightly more realistic basic states are generally not known. The aim of the CRW perspective on baroclinic instability is to predict the qualitative behaviour of perturbations to a given basic state without prior calculation of the normal modes. This section uses CRW theory to estimate the dependence of the CRW phase speeds and interaction coefficient on the wavelength. These are used to explain the normal-mode dispersion relation across the Charney range.

In the last section, it has already been assumed that the lower CRW is dominated by the surface potential-temperature wave, giving its phase speed $c_{1}^{1} \approx 2 r$. There is clearly a continuous change in the structure of the upper CRW across the Charney range from a PV $\delta$-function (located at $z=2 r$ ) to an exponential. However, the simplest argument for Charney-range behaviour is obtained by assuming that the upper-CRW shape does not change but its spread increases in proportion to wavelength. Here, the upper-CRW PV structure will be approximated by $q_{2}=Q_{2} \sin (m z)$ with vertical wave number, $m$, such that $m / k=N / f_{0}$ (i.e. $m / k=1$ when the horizontal and vertical axes are nondimensionalized by $L$ and $H$, respectively). Using PV definition (20), one finds that the counter-propagation rate of this upper wave $\gamma_{2}^{2} / k=\beta /\left(2 k^{2}\right)$ as is the case for a free Rossby wave on a $\beta$-plane. Under such approximations the upper Rossby wave speed $c_{2}^{2}=z_{2}-2 r^{2}$ (or $\Lambda z_{2}-\beta /\left(2 k^{2}\right)$ in dimensional units), where $z_{2}$ is the upper-CRW home-base (undefined at this stage).

The meridional wind induced by the lower CRW at level $z_{2}$ can be obtained from (A.1) and (A.2b), giving $v_{1}^{2}=\Theta \mathrm{e}^{-z_{2} /(2 r)}$. The interaction coefficient (nondimensionalized) obtained from (6) is $\gamma_{1}^{2}=\left\{\Theta / q_{2}\left(z_{2}\right)\right\} \mathrm{e}^{-z_{2} /(2 r)}$. Using the sinusoidal structure approximation for the upper CRW, (A.3) and (6) give $\gamma_{2}^{1}=-\left\{q_{2}\left(z_{2}\right) / \Theta\right\} r$. Since normalization (11) yields $\sigma=\left(-\gamma_{2}^{1} \gamma_{1}^{2}\right)^{1 / 2}$ then, under such approximations, the interaction strength $\sigma=\left\{r \mathrm{e}^{-z_{2} /(2 r)}\right\}^{1 / 2}$.

Figure 6 compares the values of $c_{1}^{1}, c_{2}^{2}$ and $\sigma$ obtained by the exact home-base method to the values obtained under these CRW structure approximations taking $z_{2}$ derived from the normal modes by the home-base method. Although the approximations above are rather crude, the similarity is remarkable.

However, this is not the whole story because no explanation has been provided for the relative phase of the CRWs when phase-locked and, hence, the normal-mode structures. Importantly, the CRWs are always in a hindering configuration for the growing modes of the Charney model $\left(\pi / 2<\epsilon_{+}<\pi\right)$. Substituting the approximations for Rossby wave speeds and their interaction coefficient into (13) one obtains

$$
\cos \epsilon_{+}=\frac{s-r-1}{2\left(r \mathrm{e}^{-s}\right)^{1 / 2}},
$$

where the parameter $s=z_{2} /(2 r)=m z_{2}$ compares the home-base height to the depth scale of the PV anomaly. Clearly the phase-locked angle is intimately tied to $z_{2}$. 


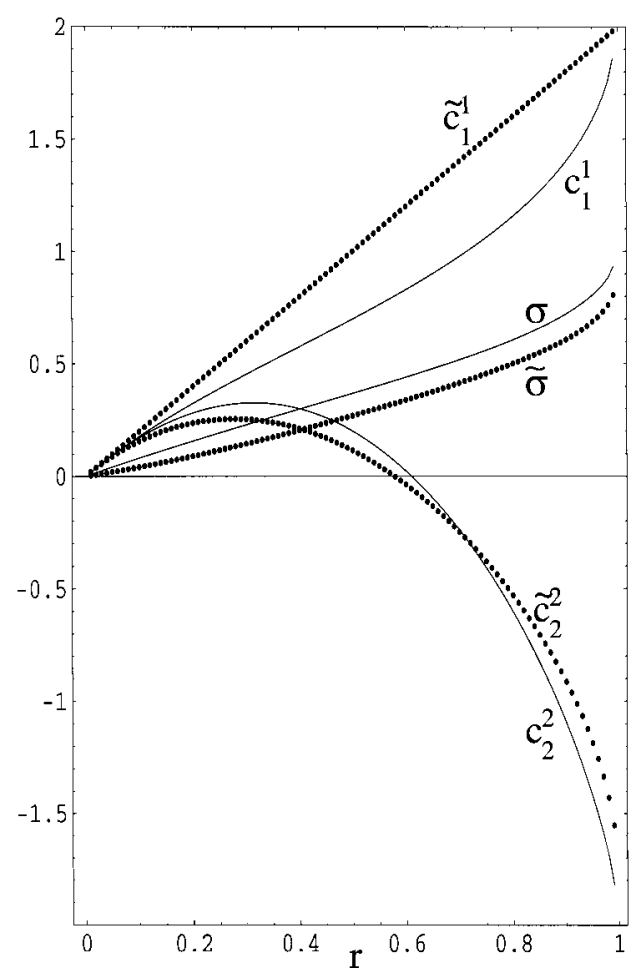

Figure 6. A comparison between the CRWs' self-induced phase speeds $\left(c_{1}^{1}\right.$ and $\left.c_{2}^{2}\right)$ and the interaction coefficient $\sigma$ obtained by the home-base method (solid lines as shown in Figs. 4(a) and (c)) and their approximate values (indicated by the dotted lines), as evaluated in section 2(c)(ii) using some simple CRW structure approximations: $\tilde{c}_{1}^{1}=2 r ; \tilde{c}_{2}^{2}=z_{2}-2 r^{2} ; \tilde{\sigma}=\left\{r \mathrm{e}^{-z_{2} /(2 r)}\right\}^{1 / 2}$.

The solution to this quadratic equation for $r^{1 / 2}$ is

$$
r^{1 / 2}=-\mathrm{e}^{-s / 2} \cos \epsilon_{+} \pm \sqrt{\mathrm{e}^{-s} \cos ^{2} \epsilon_{+}+s-1} .
$$

Taking $r^{1 / 2} \geqslant 0$, the solution can only be satisfied unconditionally if $\cos \epsilon_{+} \leqslant 0$. In other words, these simple structure CRWs must always be hindering. Furthermore, (25) then gives $s \leqslant 1+r$, providing an upper bound on the upper-CRW's height.

A lower bound on the upper-CRW's height is provided by the condition $\cos \epsilon_{+} \geqslant$ -1 . When the exponential in (25) can be linearized under a "close-interaction approximation' $s / 2 \ll 1$ (approximately satisfied over the Charney range $0 \leqslant r \leqslant 1$ ), the lower bound is found to be $s \geqslant 1-r^{1 / 2}$.

Now consider the vanishing-wavelength limit $r \rightarrow 0$. The bounds suggest that $s \rightarrow 1$, for any hindering configuration. In words, the upper CRW height decreases towards the surface in proportion to the decrease in wavelength in order to maintain interaction with the surface temperature wave for short wavelengths. Since $s \rightarrow 1$ as $r \rightarrow 0$, (25) shows that $\cos \epsilon_{+} \rightarrow 0$, which implies maximal growth but no change to CRW phase speeds through interaction. Therefore, the height of the upper CRW must approach the steering level, as observed earlier for the calculated CRW structures.

At the opposite end of the Charney range $r \rightarrow 1$, if the 'close-interaction approximation' is assumed a priori (i.e. $\mathrm{e}^{-s / 2} \approx 1-s / 2$ ), it can be deduced from (25) that 
$\cos \epsilon_{+} \rightarrow-1$ as $r \rightarrow 1$ (for any $s<2$ ). This is indeed the limiting behaviour observed for the CRWs in the Charney range (see Fig. 4(a) or (d)).

The hindering behaviour can be understood naturally from the CRW perspective. As $r$ increases, the eastward propagation rate of the lower CRW increases $(\approx 2 r)$. However, the westward counter-propagation of the upper CRW increases more rapidly $\left(\approx 2 r^{2}\right)$. The Rossby height scale also increases in proportion to $r$ and, therefore, the influence of the lower CRW extends into the domain where the zonal wind is stronger. However, since the wind only increases linearly with height, the upward shift in wave activity to faster westerlies is not sufficient to offset the increase in counter-propagation. The only way for the CRWs to phase-lock is to hinder each other's counter-propagation through interaction. The end result approaching the neutral point is a totally hindering configuration where mutual growth is no longer possible (the CRWs are in antiphase). This accounts for the maximum in normal-mode growth rate within the range $0<r<1$ even though the interaction coefficient increases across the range.

In addition, the normal-mode phase speed is the average of the two CRW wave speeds (15) so that, under the structure approximations, $c_{\mathrm{r}}=s r-r^{2}+r$. This must attain a maximum as the westward counter-propagation of the upper CRW gains importance, provided that $\mathrm{d} s / \mathrm{d} r<0$ and $s<1$. Taking the lower bound on $s=1-r^{1 / 2}$, the maximum in $c_{\mathrm{r}}$ occurs at $r \approx 0.48$, close to the observed location of the maximum in Fig. 4(b). The steering level obviously decreases beyond this maximum to zero at the neutral point.

\section{(d) The Green branches}

Interaction of the simplest relevant CRW structures, a surface temperature wave and an interior Rossby wave, has been shown to describe the behaviour of the Charney branch to a good approximation, and to account for the existence of the first neutral point and branch of westward propagating neutral modes. The main point is that the wind induced by interior PV anomalies increases with $r$ but the wind induced by surface temperature does not. The unstable Green branches and the other neutral branches can only exist through a change in the interior structure of the modes.

In section 4(b) it was shown that at the second neutral point, $r=2$, the meridional wind at the surface vanishes due to an exact cancellation of the contributions from the surface temperature wave and interior PV. This can only occur for $r>1$ because the PV structure contains a change in sign and the positive PV above $z=4$ (Fig. 3(d)) partially cancels the contribution from the lower negative PV chunk $(0<z<4)$ in the integration to obtain the surface wind. As $r$ increases above two, for structures with one sign change, the contribution from the interior PV again dominates, resulting in the westward propagating neutral-2 branch.

The Green-1 range, $1<r<2$, is now examined. The behaviour of the CRWs across the Green-1 range mimics the behaviour in the Charney range. The only difference is that both the upper and lower CRWs have another sign change in the PV structure at upper levels. The upper-level PV chunk acts to partially cancel the lower PV structure in the inversion to obtain velocity at the two home-bases. Robinson (1989) deduced, from the attribution of PV fluxes, that 'the upper lobe of the Green mode is parasitic on the mutually reinforcing interaction between the surface and the steering level that drives the instability'. Importantly, the extreme counter-propagation of both CRWs, expected as the wavelength increases, is reduced by the upper PV chunks enabling phase-locking to occur.

Towards the lower end of this range $(r \rightarrow 1)$ the upper CRW resembles a PV $\delta$ function that approaches the ground in proportion to the decrease in $r$. However, unlike 


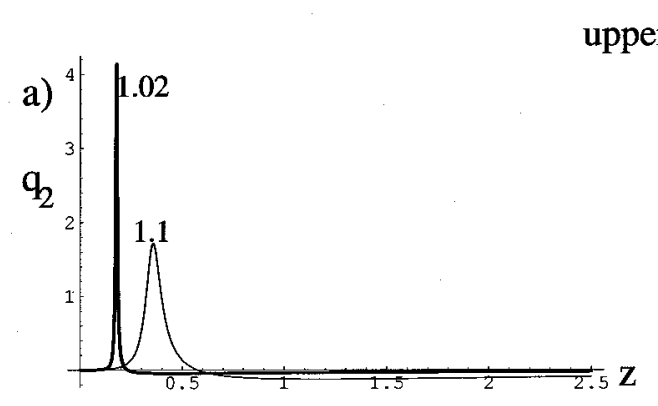

upper CRWs

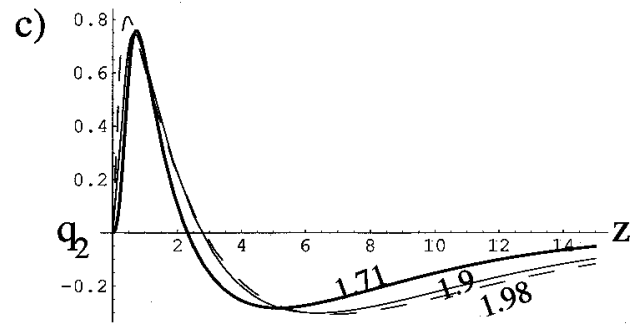

lower CRWs

b)

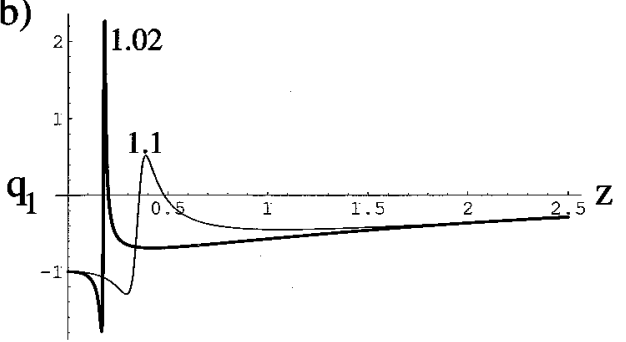

d)

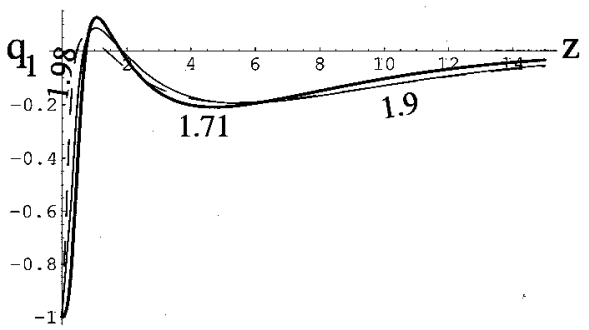

Figure 7. The vertical structures of the upper and lower CRWs, as in Fig. 5 but for the CRWs from the first Green branch. $r=1.02$ and 1.1 in (a) and (b) and $r=1.71,1.9$ and 1.98 in (c) and (d) $(r=1.71$ is the fastest-growing mode in the first Green branch).

the Charney range, it possesses a long negative tail (see Fig. 7(a)) which acts to reduce its counter-propagation rate towards zero. The lower CRW has very similar behaviour to the short-wavelength limit of the Charney range, becoming dominated by surface temperature, except that it again has a long tail (see Fig. 7(b)) acting to oppose the eastward propagation of a pure surface temperature wave.

As $r$ increases the CRWs must hinder each other increasingly in order to maintain phase-locking, and all the arguments of the last section apply. Towards the upper end of the range $(r \rightarrow 2)$, the PV maximum of the upper wave reaches a peak height and then rapidly collapses towards the ground (for $r>1.9$ ). The CRWs again tend towards a maximally hindering configuration which corresponds to antiphase. Thus, the growing normal-mode structure for $r=1.98$ (Fig. 3(d)) can be approximately obtained by subtracting the upper-CRW's PV (Fig. 7(c)) from the lower-CRW's PV (Fig. 7(d)). In the limit, the lower CRW becomes a pure surface temperature wave and the upper CRW describes all the interior PV of the neutral mode at $r=2$. There is a smooth transition from the CRW structures of Green-1 into the surface temperature and interior PV contributions of the neutral-2 branch.

Figure 8 shows the Rossby wave speeds, their phase difference, the height of the upper home-base and the interaction coefficient for the Green-1 range. The similarity to the results for the Charney range (Figs. 4(a), (b) and (c)) is striking. Note how the upperlevel PV chunks, necessary to reduce the counter-propagation rate of each CRW, also result in a reduction of the induced velocity at the other's home-base and, consequently, a reduction in the interaction coefficient, $\sigma$, compared with the Charney range. This also 
a)

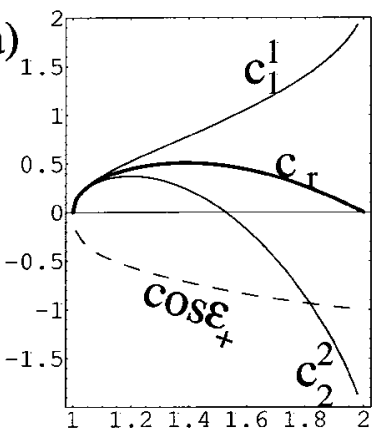

1 b)

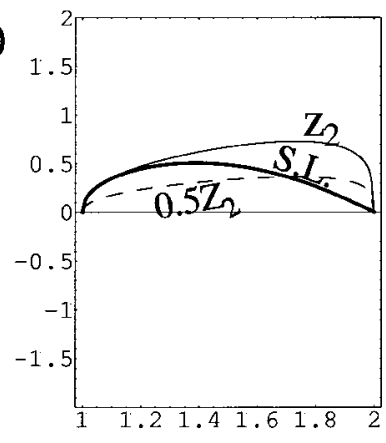

$\mathbf{r}$ c)

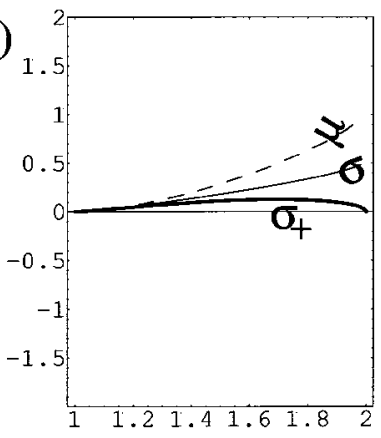

$\mathbf{r}$

Figure 8. The normal mode and associated CRW properties, as in Fig. 4(a), (b) and (c) but for the first Green branch $(1<r<2)$.

accounts for the reduction in normal-mode growth rates for each Green branch (Fig. 1) even though the phase speed increases slightly.

The results in Fig. 8 were based on the home-base analysis, rather than the more objective orthogonality method, because the CRWs obtained by the orthogonality method are strongly affected by the upper-level chunks of PV whose sole function is to counteract the increase in counter-propagation associated with increasing scale. These additional upper PV extrema affect the home-base wind of the upper CRW, $\overline{U^{2}}$, which increases dramatically between the Charney and the Green-1 ranges, making comparison between the ranges difficult. By design, for the home-base method the upper wave was defined to have zero displacement at the ground (and, therefore, positive pseudomomentum), thus determining its structure. The zero-PV line for the lower CRW was chosen to coincide with the lowest interior PV maximum of the upper CRW, located just above the steering level. The prescription of these home-bases showed more clearly than orthogonality that the growth mechanism for both the Green and Charney ranges entails the interaction between a modified edge wave and PV anomalies centred just above the steering level.

\section{CONCLUSiOnS}

It has been shown in this paper how all of the branches of the Charney model normal-mode solutions can be understood in terms of the same basic dynamical mechanisms. The essential arguments involve the Rossby-wave propagation mechanism, the scale-dependence of PV inversion for boundary and interior PV anomalies and the interaction between pairs of CRWs. The main insights are summarized below.

Existence of neutral points. The surface meridional wind induced by a surface temperature wave, $v_{\theta}(0)$, does not depend upon wavelength, $r$. However, the farfield influence of the surface wave decays exponentially from the boundary with the Rossby height scale, which is proportional to wavelength. This velocity can induce, by advection, an interior Rossby wave with similar exponential structure in PV. The integrated effect of such a PV structure on the surface wind is also proportional to wavelength. Since the interior and boundary PV gradients oppose each other, the first neutral point occurs when $v_{q}(0)$ induced by interior PV anomalies becomes sufficiently strong to cancel $v_{\theta}(0)$ exactly. The height scale used for non-dimensionalization, $H$, 
expresses the depth over which the interior PV gradient must be integrated in order to match the magnitude of the boundary PV gradient in (17). In the constant-density case, the neutral point $r=1$ corresponds to a Rossby height $k^{-1} f_{0} / \mathrm{N}=2 \mathrm{H}$.

$C R W$ description of the Charney branch. For $r<1$, surface temperature dominates the integrated effect of interior PV in a layer closest to the ground, giving eastward propagation there and a steering level above ground, resulting in instability (Bretherton 1966a). The unstable Charney branch $0<r<1$ can be described using CRW pairs obtained (for each $r$ ) from the growing normal mode and its decaying conjugate. By construction, using the home-base method, each upper CRW has no surface temperature (or PV) signature and an interior PV peak at level $z_{2}$ (its home-base). The lower CRW contains the signature of the normal mode at the surface (its home-base) and, by construction, zero PV at $z_{2}$. The lower CRW possesses low-level PV that partially opposes surface temperature in inversion. The CRW equations (4) and (5) are obtained from the PV equation at the two home-bases. Phase diagrams for CRW evolution (e.g. Fig. 3 of Part I) describe the evolution of the discrete spectrum from any initial conditions composed from a growing normal mode and its decaying complex conjugate. The phase diagram for the Charney modes is very similar to that obtained from the Eady model or barotropic Rayleigh model, highlighting the similarity in growth mechanism for all these basic states.

The short-wavelength limit. The Eady model cannot sustain growth at short wavelengths because CRWs exist only on the boundaries and, when their separation exceeds the Rossby height scale, interaction between the CRWs is too weak to maintain phaselocking against the shear (Bretherton 1966b). In contrast, instability occurs at short wavelengths in the Charney model because Rossby waves can exist at any level on the interior PV gradient. For $r \ll 1$ the upper CRW resembles an interior PV $\delta$-function and the lower CRW is dominated by surface temperature (boundary PV). The lower CRW (a Rossby edge wave) propagates at speed $2 r$ but the upper CRW cannot propagate against the flow at its home-base $\left(z_{2}\right)$. Sustained growth only occurs when $U\left(z_{2}\right)$ matches the lower CRW speed and, therefore, the upper CRW must lie on the steering level, $z_{2} \approx 2 r$. This situation was described as critical layer instability by Bretherton (1966a).

Smooth transition from the Charney branch into the neutral-1 branch. As $r \rightarrow 1$ the CRWs approach antiphase. The growing normal mode is, therefore, obtained by subtracting the upper from the lower CRW, resulting in an untilted structure, since each CRW is untilted by definition. An untilted PV structure must decay exponentially with height and take the opposite sign to surface temperature, if formed through advection by $v_{\theta}(z)$. Therefore, in the limit $r \rightarrow 1$, the lower CRW becomes a pure surface temperature wave and the upper CRW takes on the exponential PV structure of the neutral mode. As $r$ increases further, interior PV dominates the boundary contribution in the inversion for $v$ at all levels, resulting in the westward propagating modes of the neutral-1 branch.

Charney-range behaviour deduced from CRW arguments. Qualitative explanations for the behaviour over the entire Charney range can be made without prior calculation of the normal modes by making basic assumptions about the structure of interacting CRWs. The upper CRW is assumed to have a single PV peak with a spread proportional to Rossby height. The lower CRW is assumed to be dominated by its surface temperature wave. Under these approximations:

(1) The only free parameter is $s=z_{2} /(2 r)$, the ratio of the height defined for the upper-CRW home-base to the Rossby height scale. The phase difference in the growingmode locking configuration, $\epsilon_{+}$, is related to $s$ by (25). 
(2) The eastward propagation of the lower CRW increases linearly with $r$, and so does the Rossby height scale. However, the westward counter-propagation rate of the upper CRW increases as $r^{2}$, and so cannot be offset by stronger eastward advection at the Rossby height because $U$ only increases linearly with height. Thus, in order to achieve phase-locking each CRW must hinder the counter-propagation of the other through interaction, becoming increasingly hindering with $r$. Therefore, $0 \geqslant \cos \epsilon_{+} \geqslant-1$.

(3) In the short-wavelength limit $r \rightarrow 0$, it is deduced that $s \rightarrow 1$ and $\cos \epsilon_{+} \rightarrow 0$. The upper CRW and steering level approach $2 r$, no hindering is required and the CRWs lock in the maximal growth configuration.

(4) As $r \rightarrow 1$ it is deduced that $\cos \epsilon_{+} \rightarrow-1$. In words, the CRWs achieve a fully hindering configuration (antiphase) which prohibits normal-mode growth (14) even though the strength of interaction is greatest at $r=1$.

Similarity of behaviour at higher neutral points. Neutral points only occur if $v_{\theta}$ can exactly cancel $v_{q}$ at the ground. This can only be achieved for $r>1$ by a change in the structure of interior PV so that partial cancellation between PV anomalies reduces their integrated effect on surface wind. The main difference between the CRWs of the Green1 and Charney branches is the addition of a long tail of negative PV at upper levels. The sole function of this tail is to reduce the velocity induced by each CRW, counteracting the scale effect of PV inversion, which in turn reduces the counter-propagation rates of both CRWs to the extent that phase-locking can occur. The CRWs approach a totally hindering configuration as $r \rightarrow 2$ and there is a smooth transition from the CRWs of the unstable Green-1 modes into the surface temperature and interior PV structure of the neutral-2 branch (see Fig. 1(b)). Geisler and Dickinson (1975) also discussed the continuity of structure between the modes of each unstable branch into a neutral branch. Geisler and Garcia (1977) noted that a node of zero $v$ amplitude approaches the ground as $r$ increases such that the surface temperature flux is exactly zero at the neutral point.

Relevance to the atmosphere. The constant-density Charney model has an unbounded increase in zonal wind and a uniform PV gradient throughout the atmosphere and, therefore, takes no account of the extratropical tropopause. When density is allowed to vary, (22) shows how all higher neutral points are eliminated when the density scale height, $H_{\rho}$, is less than the PV-gradient scale height, $H$. The first neutral point for $H / H_{\rho}=2$ occurs at $r \approx 0.45$ which, using the scaling of section $1(\mathrm{~b})$, corresponds to a wavelength of $9500 \mathrm{~km}$ or a mid-latitude zonal wave number between one and two. In addition, Geisler and Garcia (1977) calculated normal modes for zonal flows on a $\beta$-plane and found that only the Charney and Green-1 ranges, distinguished by their vertical structure, remained when the wind profile was changed from linear to one based on climatology for the mid-latitudes. Although Geisler and Dickinson (1975) found both external and internal neutral modes for the same wind profile, Held et al. (1985) found that only the external mode exists given realistic tropospheric wind shear. The most important aspect is the stratospheric minimum in $U$ above the mid-latitude jet which traps the planetary waves and eliminates the possibility of upper-level PV structure essential to the existence of the internal neutral modes. Therefore, it can be argued that only the Charney and neutral-1 branches are relevant to the atmosphere because they have short enough wavelengths and can exist, given realistic wind profiles. The transition from unstable synoptic-scale waves to neutral planetary waves appears to be related to the CRW arguments for behaviour around the first neutral point of the Charney model.

The Eady model, which has PV gradients concentrated at the ground and the tropopause but assumes zero PV gradient elsewhere, has often been used to describe baroclinic development in the atmosphere because it neatly encapsulates the mechanism 
for baroclinic growth involving interaction between CRWs at these two levels. However, given that baroclinic growth can also be viewed as the interaction between two CRWs when the PV gradient is non-zero throughout the troposphere, and that the upper CRW is not necessarily focused at the tropopause, it is clear that the Eady model is not always appropriate. As the wavelength decreases, the upper CRW can propagate closer to the ground on the tropospheric PV gradient; short-waves are not neutralized because the upper CRW at the tropopause is too far above ground to interact, as in the Eady model.

In addition, the Eady model does not exhibit neutral modes at wavelengths longer than the fastest-growing mode. It is easy to imagine that the addition of a tropopause to the Charney basic state would result in growing modes that involve a mixture of Charney-range behaviour (for Rossby heights much less that tropopause height) and Eady-model behaviour (at longer wavelengths), as discussed for example by Held (1978). However, Robinson (1989) has shown how the large PV anomalies concentrating at the tropopause, when added to the Charney model, contribute little to boundary temperature fluxes. Interaction between surface temperature and PV anomalies just above the steering level continue to dominate the baroclinic growth mechanism. In addition, the non-zero tropospheric PV gradient enables the existence of neutral external modes with the roughly exponential PV structure of the Charney neutral-1 modes, but with an additional PV spike at the tropopause. This behaviour is indeed observed to occur for realistic baroclinic jets on the sphere (Methven et al., personal communication).

Therefore, the Charney model's dispersion relation across the Charney-range and neutral-1 branch appears more relevant to the atmosphere than that of the Eady model. Eady modes would provide a better analogy to atmospheric behaviour if the PV is homogenized on isentropic surfaces throughout the troposphere by baroclinic eddies (as argued by Sun and Lindzen (1994)). However, recently Zurita and Lindzen (2001) have shown that Charney short waves $(r<0.5)$ homogenize PV only near their steering level, since air-parcel displacements are greatest there, without removing surface temperature gradients or interior PV gradients at other levels. Longer waves can only mix interior PV to the same extent that surface temperature gradients are reduced by mixing. Since the equatorward surface temperature gradient is maintained, they argue that PV cannot be homogenized throughout the troposphere. However, where the tropopause is lower, as in a planetary-scale trough, it may lie sufficiently close to the steering level of fast-growing disturbances that Eady- and Charney-mode behaviours are indistinguishable.

Throughout this paper, the home-base method was central to the discussion because it reduces baroclinic growth on a general zonal state to the consideration of PV waves at only two locations, with interaction determined by the meridional wind induced by each wave at these locations. This provides a quantitative basis for the PV-thinking perspective on baroclinic growth put forward by Bretherton (1966b) and Hoskins et al. (1985). It is important to note that the wave structures can be obtained objectively by the orthogonality method without choosing home-base locations beforehand. Once these CRW structures are found, it is possible to identify the home-base of the upper CRW with the zero-PV line of the lower CRW. However, since only the boundary contribution to PV gradient is negative, the lower-CRW's home-base has to be identified with the ground. If the upper CRW obtained from orthogonality has zero PV at the ground, the interaction coefficients for the CRW evolution equations can be determined by the induced wind at these home-bases using (6). However, the upper CRW is generally non-zero at the ground, so that the PV-thinking description is not exact for the orthogonal CRW structures - the interaction coefficients are, instead, given by the global integrals (7). 
Nevertheless, across the Charney range, the CRW phases and structures obtained by prescription of home-bases at the ground and at the PV maximum of the resultant upper CRW almost match those from the orthogonality method. Their phase difference alone determines the Rossby wave speeds and interaction coefficient from the normalmode properties. Therefore, the PV-thinking description of baroclinic development also applies to a good approximation to the orthogonal CRWs.

In order to apply the home-base method to realistic zonal jets, one would have to choose a latitude as well as an altitude for both home-bases and the method becomes too subjective. Methven et al. (personal communication) have shown how the CRW evolution equations and composition from normal modes by the orthogonality method are generalized to the primitive equations framework on a sphere. They have shown how the CRW arguments can be applied to describe the linear dynamics of disturbances to realistic jets and explain the salient features of the normal-mode structures. Some aspects of the nonlinear baroclinic-wave development can also be related to the properties of CRWs obtained from linear theory.

\section{ACKNOWLEDGEMENTS}

Craig Bishop acknowledges support from National Science Foundation grants ATM-96-12502 and ATM-98-14376. Support from the Office of Naval Research grant N00014-00-1-0106 and Program Element 0601153N Project number BE-0333-0345 are also gratefully acknowledged. John Methven is grateful for an Advanced Fellowship sponsored jointly by the Natural Environment Research Council and the Environment Agency. All authors thank the two anonymous referees for their helpful reviews.

\section{APPENDIX}

\section{The Green function representation of $P V$}

The meridional wind induced by the PV structure $Q_{k}(z)$ from (19) can be partitioned into the meridional wind $v_{q}(z)$, induced by the interior $\mathrm{PV}, Q_{\text {int }}$, and $v_{\theta}(z)$, induced by the surface buoyancy $\Theta$. Non-dimensionalizing $v$ by $\Lambda H, Q$ by $\Lambda H / L$ and $\Theta$ by $f_{0} \Lambda L$ and using a Green function formalism (see Bishop and Heifetz 2000) it can be shown that

$$
v(z)=v_{q}(z)+v_{\theta}(z)=\int_{z^{\prime}=0^{+}}^{\infty} Q_{\text {int }}\left(z^{\prime}\right) G_{q}\left(z^{\prime}, z\right) \mathrm{d} z^{\prime}+\Theta G_{\theta}(0, z),
$$

where the Green functions are

$$
G_{q}\left(z^{\prime}, z\right)=\left\{\begin{array}{ll}
\mathrm{e}^{-k z^{\prime}} \cosh (k z) & \text { for } z<z^{\prime} \\
\mathrm{e}^{-k z} \cosh \left(k z^{\prime}\right) & \text { for } z>z^{\prime}
\end{array}, \quad G_{\theta}(0, z)=\mathrm{e}^{-k z}\right.
$$

Hence, the surface meridional wind induced by interior PV and surface buoyancy is given by:

$$
v(0)=v_{q}(0)+v_{\theta}(0)=\int_{z^{\prime}=0^{+}}^{\infty} Q_{\text {int }}\left(z^{\prime}\right) \mathrm{e}^{-k z^{\prime}} \mathrm{d} z^{\prime}+\Theta
$$


Bishop, C. H. and Heifetz, E.

Burger, A. P.

Bretherton, F. P.

Charney, J. G.

Charney, J. G. and Stern, M. E.

Davies, H. C. and Bishop, C. H.

Eady, E. T.

Fjørtoft, R.

Geisler, J. E. and Dickinson, R. E.

Geisler, J. E. and Garcia, R. R.

Gill, A. E.

Green, J. S. A.

Heifetz, E., Bishop, C. H., Hoskins, B. J. and Methven J.

Held, I. M.

Held, I. M., Panetta, R. L. and Pierrehumbert, R. T.

Hoskins, B. J., McIntyre, M. E. and 1985 Robertson, A. W.

Kuo, H. L.

Lindzen, R. S. and Tung, K. K.

Lindzen, R. S., Farrell, B. F. and Tung, K. K.

Miles, J. W.

Pedlosky, J.

Rayleigh, Lord

Robinson, W. A.

Sun, D.-Z. and Lindzen, R. S.

Zurita, P. and Lindzen, R. S.

\section{REFERENCES}

1978

1985

2000

1962

$1966 \mathrm{a}$

$1966 b$

1947

1962

1994

1949

1951

1975

1977

1982

1960

2004

1985

1973

1978

1980

1964

1987

1880

1989

1994

2001
Apparent absolute instability and the continuous spectrum. J. Atmos. Sci., 57, 3592-3608

On the non-existence of critical wavelengths in a continuous baroclinic stability problem. J. Atmos. Sci., 19, 31-38

Critical layer instability in baroclinic flows. Q. J. R. Meteorol. Soc., 92, 325-334

Baroclinic instability and the short wave cut-off in terms of potential vorticity. Q. J. R. Meteorol. Soc., 92, 335-345

The dynamics of long waves in a baroclinic westerly current. J. Meteorol, 4, 135-163

On the stability of internal baroclinic jet in a rotating atmosphere. J. Atmos. Sci., 19, 159-172

Eady edge waves and rapid development. J. Atmos. Sci., 51, 19301946

Long waves and cyclone waves. Tellus, 1, 33-52

'Stability properties of large-scale atmospheric disturbances'. Pp. 454-463 in Compendium of Meteorology, American Meteorological Society, Boston, USA

External Rossby modes on a $\beta$-plane with realistic vertical wind shear. J. Atmos. Sci., 32, 2082-2093

Baroclinic instability at long wavelengths on a $\beta$-plane. J. Atmos. Sci., 34, 311-321

Atmosphere-ocean dynamics. Academic Press

A problem in baroclinic instability. Q. J. R. Meteorol. Soc, 86, 237-251

The counter-propagating Rossby-wave perspective on baroclinic instability. I: Mathematical basis. Q. J. R. Meteorol. Soc., 130, 211-231

The vertical scale of an unstable baroclinic wave and its importance for eddy heat flux parameterizations. J. Atmos. Sci., 35, 572-576

Pseudomomentum and the orthogonality of modes in shear flow. J. Atmos. Sci., 42, 2280-2288

Stationary external Rossby waves in vertical shear. J. Atmos. Sci., 42, 865-883

On the use and significance of isentropic potential vorticity maps. Q. J. R. Meteorol. Soc., 111, 877-946

Dynamics of quasi-geostrophic flows and instability theory. $A d v$. Appl. Mech., 13, 247-330

Wave overreflection and shear instability. J. Atmos. Sci., 35, 16261632

The concept of wave overreflection and its application to baroclinic instability. J. Atmos. Sci., 37, 44-63

A note on Charney's model of zonal-wind instability. J. Atmos. Sci., 21, 451-452

Geophysical Fluid Dynamics, Second Edition. Springer-Verlag

On the stability, or instability, of certain fluid motions. Proc. London Math. Soc., 9, 57-70

On the structure of potential vorticity in baroclinic instability. Tellus, 41A, 275-284

A PV view of the zonal mean distribution of temperature and wind in the extratropical troposphere. J. Atmos. Sci., 51, 757-772

The equilibration of short Charney waves: Implications for PV homogenization in the extratropical troposphere. J. Atmos. Sci., 58, 3443-3462 